\title{
Validation for Downstream Computer Aided Manufacturing and Coordinate Metrology Processes
}

\author{
Asa Trainer \\ International TechneGroup Incorporated
}

This publication is available free of charge from:

http://dx.doi.org/10.6028/NIST.GCR.16-003

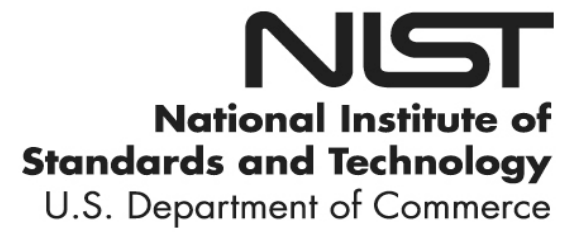




\title{
Validation for Downstream Computer Aided Manufacturing and Coordinate Metrology Processes
}

\author{
Prepared for \\ Allison Barnard Feeney \\ Thomas Hedberg, Jr. \\ U.S. Department of Commerce \\ Engineering Laboratory \\ National Institute of Standards and Technology \\ Gaithersburg, MD 20899-8260

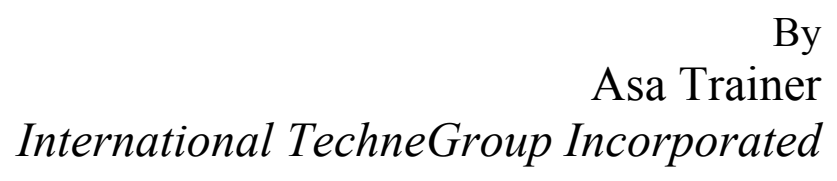

This publication is available free of charge from:

http://dx.doi.org/10.6028/NIST.GCR.16-003

September 2015

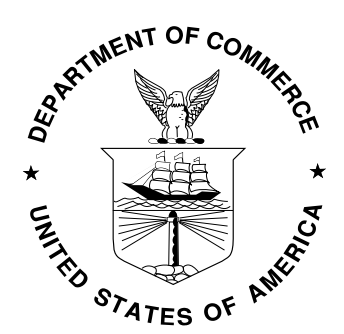

U.S. Department of Commerce

Penny Pritzker, Secretary

National Institute of Standards and Technology Willie May, Under Secretary of Commerce for Standards and Technology and Director 


\section{DISCLAIMERS}

This report was prepared for the Engineering Laboratory of the National Institute of Standards and Technology (NIST) under the following cooperative agreement:

- 70NANB14H256, International TechneGroup Incorporated, "Validation for Downstream Computer-Aided Manufacturing and Coordinate-Metrology Processes"

Any opinions, findings, conclusions, or recommendations expressed in this publication do not necessarily reflect the views of NIST. Additionally, neither NIST nor any of its employees make any warranty, expressed or implied, nor assume any legal liability or responsibility for the accuracy, completeness, or usefulness of any information, product, or process included in this publication.

This paper may identify certain commercial systems. Such identification does not imply recommendation or endorsement by NIST. Nor does it imply that the products identified are necessarily the best available for the purpose. 


\title{
Validation for Downstream Computer-Aided Manufacturing and Coordinate-Metrology Processes \%
}

\author{
Semi-Annual Technical Report \\ Reporting Period - April 1, 2015 to September 30, 2015
}

\begin{abstract}
To achieve industry's vision of the Model-Based Enterprise (MBE), the MBE strategy must include model-centric data interoperability for design to manufacturing and quality in the supply chain. The Model-Based Definition (MBD) is created by the OEM using Computer-Aided Design (CAD) tools. This information is then shared with the supplier so that they can manufacture and inspect the physical parts. Today, almost all suppliers use Computer-Aided Manufacturing (CAM) and Coordinate Measuring Machine (CMM) models respectively for these tasks. In the MBE vision for model-centric data exchange, the CAD model must include product and manufacturing information (PMI) in addition to the shape geometry. Today's CAD tools can generate models with embedded PMI, with the emergence of STEP AP242, a standards-based model with embedded PMI can now be shared downstream. This project investigates the ability to utilize a STEP AP242 model with embedded PMI for CAD-to-CMM data exchange. The project examines transformational processes and identifies gaps that will impact industry's ability to achieve model-centric data interoperability cost-effectively in the pursuit of the MBE vision.
\end{abstract}




\section{TABLE OF CONTENTS}

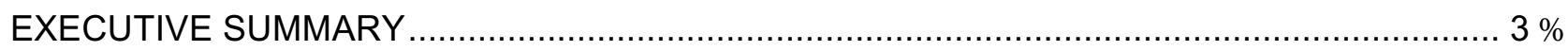

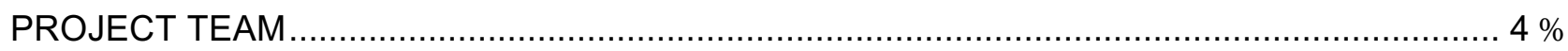

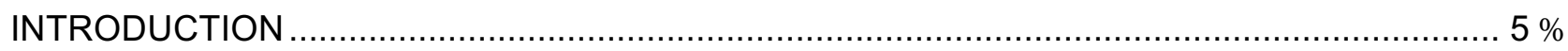

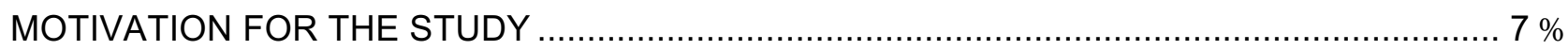

METHOD

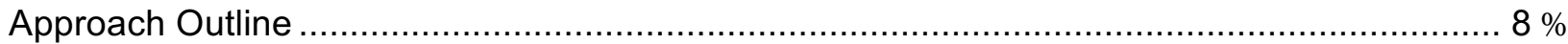

Determination of Test Cases - Models and Data Sets...................................................... $9 \%$

Definition of Validation Metrics …………………….................................................. $9 \%$

Development of CAM/CM (ACIS) Validation Algorithm .................................................. $10 \%$

Validation of Extended ACIS and Collection and Analysis of Validation Metrics .................. $10 \%$

Scope and Mapping Process for PMI Mapping to QIF ................................................. $11 \%$

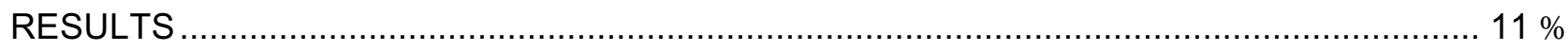

Results from Mapping PMI between STEP and QIF .................................................. $11 \%$

Results from Validation of Original Project Test Models.................................................. $12 \%$

Results from Validation of Test Models from NIST Data Sets ............................................. $14 \%$

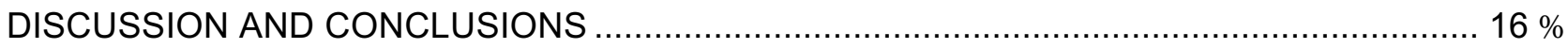

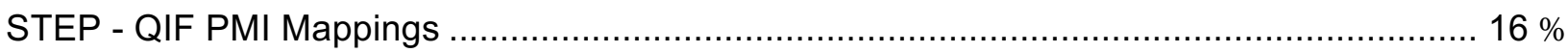

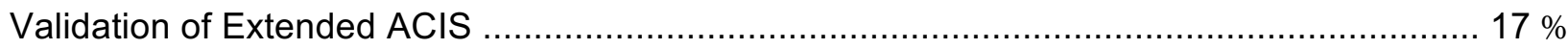

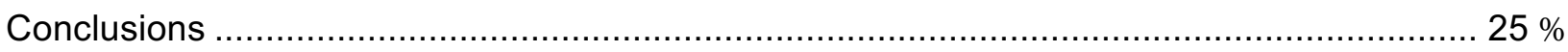

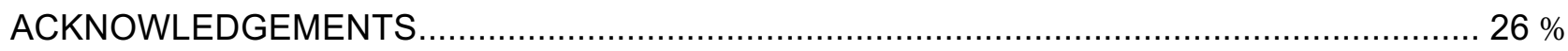

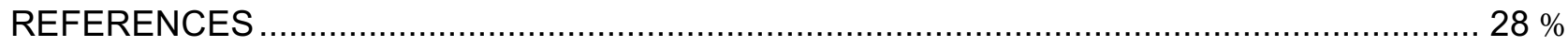

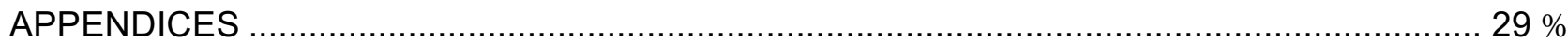

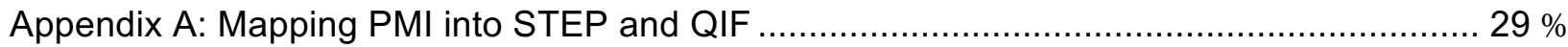

Appendix A.1: Mapping GD\&T into STEP and QIF ................................................. $29 \%$

Appendix A.2: Mapping Linkages into STEP and QIF ……....................................... $38 \%$

Appendix A.3: Mapping PMI Features and User Defined Tolerances into STEP and QIF . $39 \%$

Appendix A.4: Mapping of Boundary Representation (BREP) Geometry into STEP and QIF \%

$41 \%$

Appendix A.5: Mapping Other MBD-related Items into STEP and QIF .......................... $42 \%$

Appendix B: Validation Results for Test Models ............................................................ $43 \%$

Appendix B.1: Validation Differences by PMI Category for Test Models .......................... $43 \%$

Appendix B.2: Percent Clean (\%) by PMI Category for Test Models ............................... $44 \%$ 


\section{EXECUTIVE SUMMARY}

To achieve industry's vision of the Model-Based Enterprise (MBE), the MBE strategy must include model-centric data interoperability for design to manufacturing and quality in the supply chain. The Model-Based Definition (MBD) is created by the OEM using Computer-Aided Design (CAD) tools. This information is then shared with the supplier so that they can manufacture and inspect the physical parts. Today, almost all suppliers use Computer-Aided Manufacturing (CAM) and Coordinate Measuring Machine (CMM) models respectively for these tasks. Until recently data for shape has been transferred downstream via STEP AP203 but collaborators had to handle product manufacturing information (PMI, i.e. dimensions and tolerances) for metrology planning and execution via transcription from drawings. With the recent availability of STEP AP242, the promise of direct transformation of design data to downstream consumers including $\mathrm{PMI}$ in a single MBD package now exists. The vision of MBE is that by using STEP AP242 for interoperability of rich design information with the manufacturing and metrology supply chain, the process would be faster, more accurate, and be less error prone.

This project was developed to investigate the interaction between STEP AP242 and CAM and CMM systems by using ACIS, the predominant modeling kernel in the manufacturing and metrology space. Algorithms were developed to transform the data but more importantly to show that validation was a critical component of success in the transformation process. In addition to examining ACIS as a vehicle for transforming design data into metrology data, the team also examined the emerging QIF standard and mapped it to STEP AP242 to determine what effort might be required to make STEP and QIF the standards of choice for this process.

The project team included interoperability experts in STEP and ACIS as well as QIF. The team developed metrics for validation of PMI data into ACIS and examined the results of the STEP to ACIS transformation and validation process. The team also examined STEP and QIF standards to determine where there was commonality, where there was overlap, and where there were gaps between the standards.

This project demonstrated the ability to transform design model data that included PMI from STEP AP242 data into extended ACIS for use by Manufacturing and Metrology systems. It confirmed that transformation of such semantically rich PMI data requires an automated method to validate that the transformed data is complete and correct for downstream use. 


\section{PROJECT TEAM}

\section{Project Coordination}

Asa Trainer, International TechneGroup Incorporated

\section{PMI Mapping}

Curtis Brown, Honeywell FMT

Victor Mikushin, Capvidia

Ed Paff, International TechneGroup Incorporated

Tom Kramer, NIST

Tomasz Luniewski, Capvidia

Daniel Campbell, Metrosage

Hui-Min Huang, NIST

\section{Validation Algorithm Development}

Ed Paff, International TechneGroup Incorporated

Dave Smith, International TechneGroup Incorporated

\section{Validation Analysis}

Doug Cheney, International TechneGroup Incorporated

\section{Research Advisors}

Allison Barnard Feeney, National Institute of Standards and Technology

Thomas Hedberg, Jr., National Institute of Standards and Technology 


\section{INTRODUCTION}

The challenge: Given industry's vision of MBE, and immerging research into modelcentric data interoperability between design and manufacturing and quality inspection, shouldn't these new model-centric data interoperability processes include validation mechanisms to ensure data quality and provide a metric for measuring the evolution of these processes as they mature?

To achieve the industry vision of the Model-Based Enterprise (MBE), the MBE strategy must include model-centric data interoperability for design to manufacturing and quality in the supply chain. The Model-Based Definition (MBD) is created by an OEM using Computer-Aided Design (CAD) tools. This information is then shared with the supplier so that they can manufacture and inspect the physical parts. Much of the supply base consists of Small and Medium Enterprise (SME) manufacturers. Today, almost all suppliers use Computer-Aided Manufacturing (CAM) and Coordinate Measuring Machine (CMM) models respectively for these tasks. Traditionally, design data is provided from the OEM to supplier in the form of full detail 2-dimensional (2D) drawings. More recently the data has also included a 3-dimensional (3D) model with shape geometry. This model is often provided in a standards-based model format - STEP AP203 is prevalent. However, in addition to geometry, the downstream CAM and CMM processes also require product and manufacturing information (PMI) in order to fabricate and inspect the physical part.

The vision of MBE and supply chain interoperability using an MBD-based process has great potential to improve efficiency and achieve higher product quality. In 2014 a new STEP Application Protocol (AP), AP242, was published which was developed to consolidate and replace previous APs and includes support for PMI (Reference A). STEP AP242 would seem to represent the way forward to solving this downstream interoperability problem.

As stated in Reference A, many downstream systems have proprietary direct translation capability and that some of those capabilities include PMI support, though none of these systems yet support the STEP standard. The long term desire, however, is for the CAM and CM communities to support the direct consumption of STEP AP242 and other international standards with precise geometry and semantic PMI data, a more achievable approach in the short term is to leverage the modeling kernels that are the basis for many CAM and CM systems, the ACIS and Parasolid kernels. The research conducted in Reference A clearly demonstrates that this standards-based approach to downstream interoperability is feasible.

While standards-based exchange provides significant benefit to industry, one challenge that must be addressed is verification and validation of translations, ensuring adequate product data quality. The ability to validate that model data is transformed properly during the interoperability process is important to manufacturers large and small. Without it, data consumers cannot be sure that their content is complete and accurate and the risk of potentially costly rework is a major concern. Performing such validation by hands-on inspection has been, 
when 2D drawings are the method for exchange of manufacturing data in the supply chain, the purview of design checkers. The need for confidence in the conformance of 3D model data to quality standards is well understood [13]. While validation of 3D modeling data by hands-on inspection is certainly possible, it is not desirable. The process is painstakingly slow, requires a high level of skill and attention to detail, and there is a high likelihood of error even when done by a skilled practitioner. It is also important to note that even though the burden of validation is important for geometry interoperability, it is even more critical and more challenging when PMI interoperability is required. As such, formal requirements for verification of model data, particularly PMI data, and validation of derivative variants of that data for collaboration purposes are now in place [14].

Automation of validation for CAD-to-CAD interoperability processes is becoming an important tool particularly for 3D model data containing PMI (Reference B and Reference C). Validation of transformed model data in the CAM and CM context is just as important to instilling confidence in automated processes for the CAM and CM communities.

STEP is generally considered to be the method of choice for CAD-to-CAD interoperability for shape, and with the availability of semantic PMI constructs in STEP AP242, STEP shows promise as a strong candidate for interoperability between CAD and CM. The metrology community has developed their own standard, the Quality Information Framework (QIF, Reference D), to support the unique needs of that community. QIF contains data structures for collecting, organizing, and analyzing measurement data and metrology systems are beginning to make use of those QIF standard structures. QIF also has its own MBD data structures for both shape (geometry) and PMI. A case could be made for interoperability between these two complementary standards.

First hypothesis: Transformation of MBD data from CAD to CAM and CAD to CMM requires a mechanism to validate the integrity and quality of the downstream data and that validation of such data provides insight into the state of art for downstream interoperability systems.

Second hypothesis: The STEP AP242 and QIF standards share common ground as far as geometry and PMI interoperability is concerned and that there is an opportunity to bridge the gap between CAD and CMM communities through interoperability between these two standards.

This project will test these two hypotheses. The first hypothesis is that model-based data transformed from CAD-to-CAM and CAD-to-CMM via STEP AP242 and ACIS can be validated using automated methods and that there is significant value in performing such validations to evaluate the nascent capabilities for interoperability between design and downstream processes. The second hypothesis is that STEP AP242 and QIF 2.0 are aligned as far as geometric and PMI data are concerned and that harmonization these standards represent a 
potential way forward for future interoperability tools and processes. The project will answer the question of the current state of evolution of early CAD-to-CMM transformation tools through demonstration and measured results. It will also answer the question of how closely aligned the two standards are that may connect CAD to CMM. It will provide for discussion and recommendations based upon the findings and observations. Finally, the project will draw conclusions about the level of maturity of standards-based model-centric data interoperability between upstream creation and downstream consumption.

\section{MOTIVATION FOR THE STUDY}

The motivation: Demonstrate the value of validation of model-centric CAD-to-CAM and CAD-to-CMM data interoperability when using STEP AP242 with embedded $P M I$ transformed into extended ACIS data.

The research team believes this project will assist industry in its drive to achieve the vision of the Model-Based Enterprise down into the manufacturing supply chain if the above hypotheses can be substantiated.

A standards-based workflow for design to manufacturing and inspection involves exchange of CAD-to-AP242-to-CAM-and-CMM models. Validation and verification of this translation process is important, especially for regulated industries. An important part of quality assurance is traceability back to the design definition. To assure compliance at any point in the manufacturing or inspection process, it is essential to have validation and verification of the models throughout the data-exchange process.

When moving to a model-based paradigm, the verification process is more complex since the goal is for the model geometry and PMI to be consumed directly by downstream software systems. Verification in this context requires each and every PMI element be analyzed for syntactical and semantic accuracy including proper association of the PMI to geometric references in the 3D geometry.

In addition to verifying that PMI content has been authored correctly, each time the data is transformed - from CAD to STEP and from STEP to CAM/CMM - the data must be validated to be sure no data corruption occurred during the transformation process. Since the information content in the 3D model is no longer in the form of a visually inspect-able 2D drawing, special software algorithms are required to perform the verification and validation processes on all but the simplest models. 


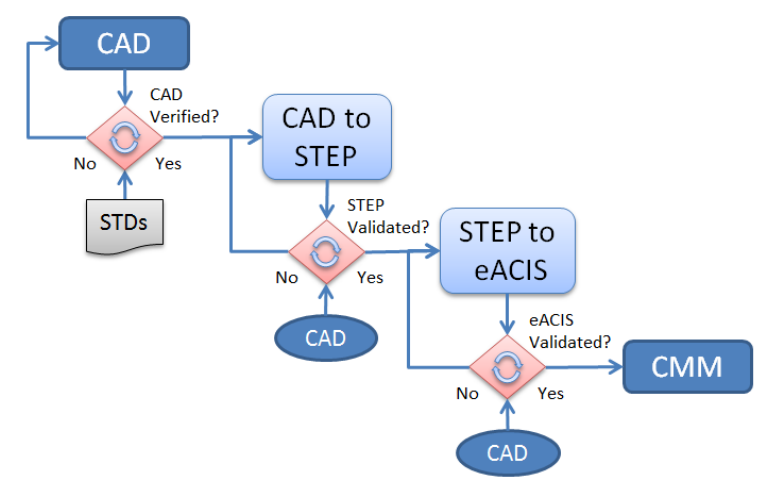

Figure 1 - Verification and validation process flow diagram

In the case of this project, we performed verification and validation following the process outlined in Figure 1. We performed verification and validation using a combination of traditional visual inspection techniques and automated techniques. In general use on more complex models, automated techniques would have been required.

The research team also believes that the developed technology and the aforementioned standards are extensible to include PMI data items beyond those used in the test models. The application can successfully exchange more complex parts than the project's demonstrated test models. The software application used in this demonstration will achieve Technology Readiness Level (TRL) 6. A list of TRL definitions is given in Appendix C. This provides motivation for commercialization of interoperability validation tools to provide standards-based 3D models with embedded PMI to the CAM and CMM industry. It is expected that CAM and CMM tools providers would be motivated to commercialize the ability to receive and utilize standards-based models with embedded PMI.

As the identified gaps in current tools and standards are addressed to achieve this level of commercialization, and the process and skill gaps are overcome by industry users, the opportunity to improve efficiency and effectiveness across the product lifecycle will be provided in design, manufacturing, inspection, and collaboration across the supply chain.

\section{METHOD}

The methodology used to test the above hypotheses is described below.

\section{Approach Outline}

To test the hypotheses, the following approach was used:

- Determine test cases for validation processing,

- Develop metrics for evaluating validation results,

- Develop prototype software for automatic validation of extended-ACIS models against original CAD data,

- Validate extended-ACIS models against source STEP model data, 
- Collect and analyze metrics data to evaluate the maturity of STEP to ACIS transformation process and also to evaluate the maturity of the validation process itself,

- Determine the scope for mapping of STEP to QIF, and

- Map model-based PMI requirements from STEP to QIF and identify gaps that inhibit development of interfaces between these two standards.

Each of these elements of the approach is discussed below.

\section{Determination of Test Cases - Models and Data Sets}

In keeping with the collaborative nature of this project and its companion project (Reference A), the initial two test cases for this project were selected to be the same two models selected by that companion project team. This was a logical choice as native NX ${ }^{\mathrm{TM}}$ CAD model data already existed and had been used as the source for the intermediate STEP models and the ACIS models in CAM and CM. Furthermore, all of the data - native, STEP, and ACIS - had been thoroughly analyzed by hand for completeness and correctness. These data were used to exercise the developed algorithms for automatically validating PMI-extended ACIS against native CAD models. These test cases are shown in Figures 2 and 3 below.
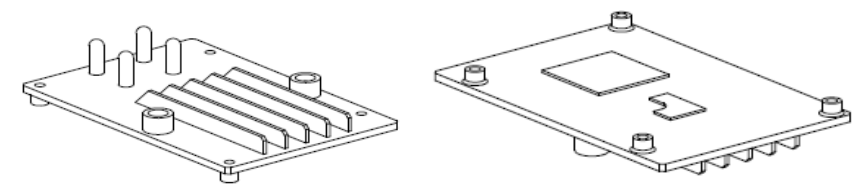

Figure 2 - Test Case \#1 - Milled Part 827-9999-904
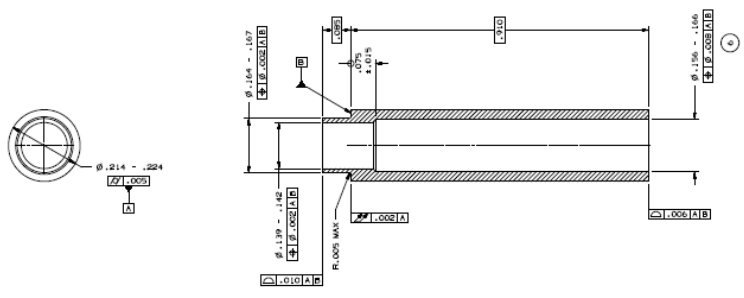

Figure 3 - Test Case \#2 - Revolved Part 827-9999-903

The above two test cases were augmented by leveraging a subset of the now well-known NIST test cases and models available from References B and C. The test data were chosen from STEP files generated during several recent PDES/ProSTEP CAx test rounds. The selected STEP models were those for which semantic PMI annotation had been generated.

\section{Definition of Validation Metrics}

A mechanism was needed to quantify the extent of successful transformation of PMI annotation information from the original CAD PMI data to that same PMI data in ACIS format. The authors selected five major categories of PMI annotation data to be used as metrics for this initial validation research activity. For each of these major categories, a selected set of semantic PMI content (parameter values) would be evaluated. 
The PMI annotation categories selected and the semantic parameters to be used for validating for each annotation type are shown Table 1 below:

Table 1 - PMI Annotation Categories for Validation Purposes

\begin{tabular}{|l|l|}
\hline PMI Category & PMI Content Validated \\
\hline Datum Feature Symbol (DFS) & Identifier \\
\hline Datum Target Symbol (DTS) & Identifier \\
& Target type \\
\hline Dimension (DIM) & Tolerance format \\
& Nominal value (with unit) \\
& Upper and lower values (with unit) \\
\hline Feature Control Frame (FCF) & Type \\
& Symbol \\
& Overall tolerance \\
& Datum reference frame identifiers \\
& Modifiers \\
\hline Note (NOTE) & Text \\
\hline - Symbols shown in parentheses above are used in the validation results table in \\
Appendix B to identify each PMI annotation category listed \\
\hline
\end{tabular}

\section{Development of CAM/CM (ACIS) Validation Algorithm}

The plan for algorithm development was split into two phases. The first phase was to focus on the development of a prototypical capability to validate ACIS geometry. Data structures for geometry in ACIS are well known, stable, and other geometry data models can easily be mapped to ACIS. Following the development of a validation capability for geometry, the second, more challenging phase would focus on development of validation capability for PMI data. This second phase was expected to be more complex. This was due to the fact that ACIS is first and foremost a geometry model schema and that extensions to include PMI constructs are evolving. Further comments on this extended ACIS, its maturity for PMI, and the effects of that maturity on validation will be discussed in the results section.

\section{Validation of Extended ACIS and Collection and Analysis of Validation Metrics}

Validation of extended ACIS was performed by applying the developed algorithm to evaluate geometry and PMI entities in both each STEP model and its derived ACIS model. This was done for the original companion project test cases as well as selected members of the broader NIST test suite. For each test case, each entity pair was analyzed for correctness of the mapping and compared for matching values of the entity's properties. The metrics described in Table 1 were counted as either "clean" or "different" for every PMI entity pair. An entity pair was considered "clean" if the entity structure properties were preserved during transformation and if the values of those properties in the target ACIS data matched the values of the properties in the source STEP data. The results were analyzed to determine, where possible, whether any differences were a result of issues in the transformation algorithm or a result of the validation algorithm. 


\section{Scope and Mapping Process for PMI Mapping to QIF}

For the STEP QIF mapping exercise, a group of STEP and QIF subject matter experts from ITI, Honeywell FM\&T, Capvidia, and Metrosage, together with STEP and QIF standards experts from NIST, gathered in a workshop at the Spring 2015 PDES Offsite in Gaithersburg, MD, to form a working group responsible for the PMI mapping task. That working group collectively determined ground rules for the mapping research and outlined a reasonable scope for the mapping process. The working group agreed to leverage the STEP and ACIS mapping tables produced as part of the companion project (again Reference A) as the framework for the QIF mapping. The working group also agreed to focus attention on the subset of QIF referred to as QIF MBD - that part of the QIF standard addressing product shape and PMI content that would likely be received from upstream design-related processes.

Following the workshop, the working group held bi-weekly conference calls over the course of a 3-month period to review mapping progress, discuss issues concerning interpretation of the standards, and formulate new work items for the next two-week period. Over the course of that period the existing STEP-ACIS mapping table was extended to include QIF MBD constructs. Additionally, gaps between the standards that could not be closed were identified for later resolution.

\section{RESULTS}

\section{Results from Mapping PMI between STEP and QIF}

A broad subset of the possible categories of PMI data were mapped as part of this project. The mapping categories [STEP -> QIF] are listed below:

-\% Boundary representation geometry,

-\% Construction geometry, including points, planes, coordinate systems, curves, surfaces, and axes,

-\% GTOLs and dimensions (including their tolerances),

-\% Default or general tolerances,

-\% Notes (including general and flag notes),

-\% Tables,

-\% Title block information,

-\% Datums, including datum targets, datum tags, and datum reference frames, and

-\% Saved views.

Certain categories of data were not mapped [STEP to QIF] as part of this project. Mapping of the three non-mapped data categories is a subject for future research. These categories are indicated in the list below:

-\% Layers and groups,

-\% Assembly structure, including component instancing, and

-\% Cross-sections (and planar sketches). 
The result of the mapping exercise was a table of PMI elements and their appropriate entities in STEP, ACIS, and QIF formats. For the purposes of discussion in this report, the table was broken up in to separate sections. Though focused on mapping of PMI related content in this study, the authors have included mappings for geometric shape entities for completeness. The various mappings are listed below:

- GD\&T Entities

- Linkage Entities

- PMI Features and User Defined Tolerances

- Boundary Representation Geometry Entities

- Other MBD-related Entities

All mapping tables are presented in Appendix A and discussed later in this report. An example of a portion of the GD\&T mapping table is shown in Figure 4 below.

\begin{tabular}{|c|c|c|c|}
\hline & STEP AP242 & ACIS & QlF \\
\hline \multicolumn{4}{|l|}{$\begin{array}{l}\text { PMI } \\
\text { dimension types }\end{array}$} \\
\hline linear dimension & dimensional_location & spaxpmi_dimension (DIMSUBTYPE_LENGTH_OR_DISTANCE) & LengthCharacteristicDefinitionType \\
\hline angular dimension & angular_location/angular_size & spaxpmi_dimension (DIMSUBTYPE_ANGLE)(no way to specify me & AngularCharacteristicDefinitionType \\
\hline radius dimension & dimensional_size & spaxpmi_dimension (DIMSUBTYPE_RADIUS) & RadiusCharacteristicDefinitionType \\
\hline $\begin{array}{l}\text { diameter dimension } \\
\text { oriented dimension }\end{array}$ & $\begin{array}{l}\text { dimensional__ize } \\
\text { oriented_dimensional_location }\end{array}$ & $\begin{array}{l}\text { spaxpmi_dimension(DIMSUBTYPE_DIAMETER) } \\
\text { not covered }\end{array}$ & $\begin{array}{l}\text { DiameterCharacteristicDefinitionType } \\
\text {... }\end{array}$ \\
\hline $\begin{array}{l}\text { curved dimension } \\
\text { coordinate dimension }\end{array}$ & dimensional_location_with_path/dimensional_size_with_path & $\begin{array}{l}\text { spaxpmi_dimension(DIMSUBTYPE_CURVILINEAR) } \\
\text { spaxpmi_dimension(DIMSUBTYPE_COORDDIM2D, DIMSUBTYPEE }\end{array}$ & $\begin{array}{l}\text { CurvedLengthCharacteristicDefinitionType } \\
\text { LinearCoordinateCharacteristicDefinitionType }\end{array}$ \\
\hline \multicolumn{4}{|c|}{ 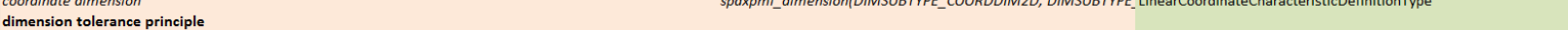 } \\
\hline independency & shape_dimension_representation.name & not covered & $\diamond$ EnvelopeRequirement(FALSE) \\
\hline \multicolumn{4}{|l|}{ dimension values } \\
\hline nominal value & measure_representation_item & dimension value & $<$ TargetValue \\
\hline nominal value with qualifier & qualified_representation_item & not covered & $>$ TargetValue \\
\hline nominal value with plus/minus bounds & plus_minus_tolerance & not covered & <>DefinedAsLimit(FALSE) \\
\hline value range & measure_representation_item & dimtol lower limit/dimtol upper limit & $\diamond$ DefinedAsLimit(TRUE) \\
\hline tolerance class & limits_and_fits & not covered & \\
\hline \multicolumn{4}{|l|}{ dimension modifiers } \\
\hline basic/theoretical & descriptive_representation_item & dimension_type (dimtype_basic) & $<>$ DimensionType(BASIC) \\
\hline reference/auxiliary & descriptive_representation_item & dimension_type (dimtype_reference) & $<$ DimensionType(REFERENCE) \\
\hline controlled radius & descriptive_representation_item & not covered & RadiusCharacteristicDefinitionType $>$ ControlledRadius(TRUE) \\
\hline square & descriptive_representation_item & not covered & SquareCharacteristicDefinitionType \\
\hline statistical tolerance & descriptive_representation_item & dimension_type (dimtype_tolerance) & CharacteristicDefinitionBase Type $>>$ StatisticalCharacteristic(TRUE) \\
\hline
\end{tabular}

Figure 4 - Example of PMI(GD\&T)/STEP/ACIS/QIF Mapping Table

\section{Results from Validation of Original Project Test Models}

Development and demonstration of a process to exchange standards-based models with embedded PMI from design to downstream systems was successful within the scope of the limited test models used in this project. The validation results, as defined by PMI element counts, for the downstream models are provided in Table 2. The validation shows that all dimensions, tolerances, and datum features were transformed and exchanged.

Indicated in Table 2, general notes could not be mapped to ACIS. Although manual validation showed correct PMI counts (for PMI other than general notes), further detailed examination by automated validation of the downstream models using analysis software found anomalies in the transformed data. 
Table 2: Validation of model transformations using embedded PMI entity count

\begin{tabular}{|c|c|c|c|c|c|c|c|c|c|c|}
\hline \multirow{2}{*}{$\begin{array}{r}\begin{array}{c}\text { PMI Elements } \\
\text { (by format) }\end{array} \\
\text { Model (827-9999) }\end{array}$} & \multicolumn{2}{|c|}{$\overline{\mathbf{N X}}$} & \multicolumn{2}{|c|}{ STEP } & \multicolumn{2}{|c|}{ ACIS } & \multicolumn{2}{|c|}{ Mastercam } & \multicolumn{2}{|c|}{ MiCAT } \\
\hline & -903 & -904 & -903 & -904 & -903 & -904 & -903 & -904 & -903 & -904 \\
\hline Dimension & 8 & 54 & 8 & 54 & 8 & 54 & 8 & 54 & 8 & 54 \\
\hline Tolerance & 6 & 13 & 6 & 13 & 6 & 13 & 6 & 13 & 6 & 13 \\
\hline Datum Feature & 2 & 3 & 2 & 3 & 2 & 3 & 2 & 3 & 2 & 3 \\
\hline Notes (not semantic data) & 7 & 8 & 7 & 8 & 0 & 0 & 7 & 8 & 0 & 0 \\
\hline Total & 23 & 78 & 23 & 78 & 16 & 70 & 23 & 78 & 13 & 68 \\
\hline
\end{tabular}

Table 3 shows the results of automated validation of model transformations. In the -904 model, the automated validation tool showed that though all dimensions were transformed and, for the most part, semantically correct, a rule violation occurred when the dimension tolerance zone for one dimension was considered large relative to its nominal value. The -903 model, like the -904 model, was flagged for an instance of this same rule violation. The -903 model was also flagged for failure to maintain the semantic definition of limit dimensions in four instances of that dimension type when transformed from STEP to ACIS.

Table 3: Validation of models using analysis software

\begin{tabular}{lllll}
\hline Model File & DFS $^{1}$ & DIM $^{2}$ & FCF $^{3}$ & Clean $^{\text {Clean }}$ \\
& Clean & Clean & Percent \\
\hline $827-9999-903$ & 2 & 3 & 6 & $69 \%$ \\
$827-9999-904$ & 3 & 53 & 13 & $99 \%$ \\
\hline
\end{tabular}

The counts shown in Table 3 refer to the number of entities that are clean (e.g., pass all syntax and semantic validity checks during analysis of STEP to ACIS transformations). The clean percent in Table 3 ignores note entity errors.

${ }^{1}$ DFS $=$ Datum Feature Symbol

${ }^{2} \mathrm{DIM}=$ Dimension

${ }^{3} \mathrm{FCF}=$ Feature Control Frame 
Example validation results are shown in Figure 5 (for semantic representation PMI) below. \%

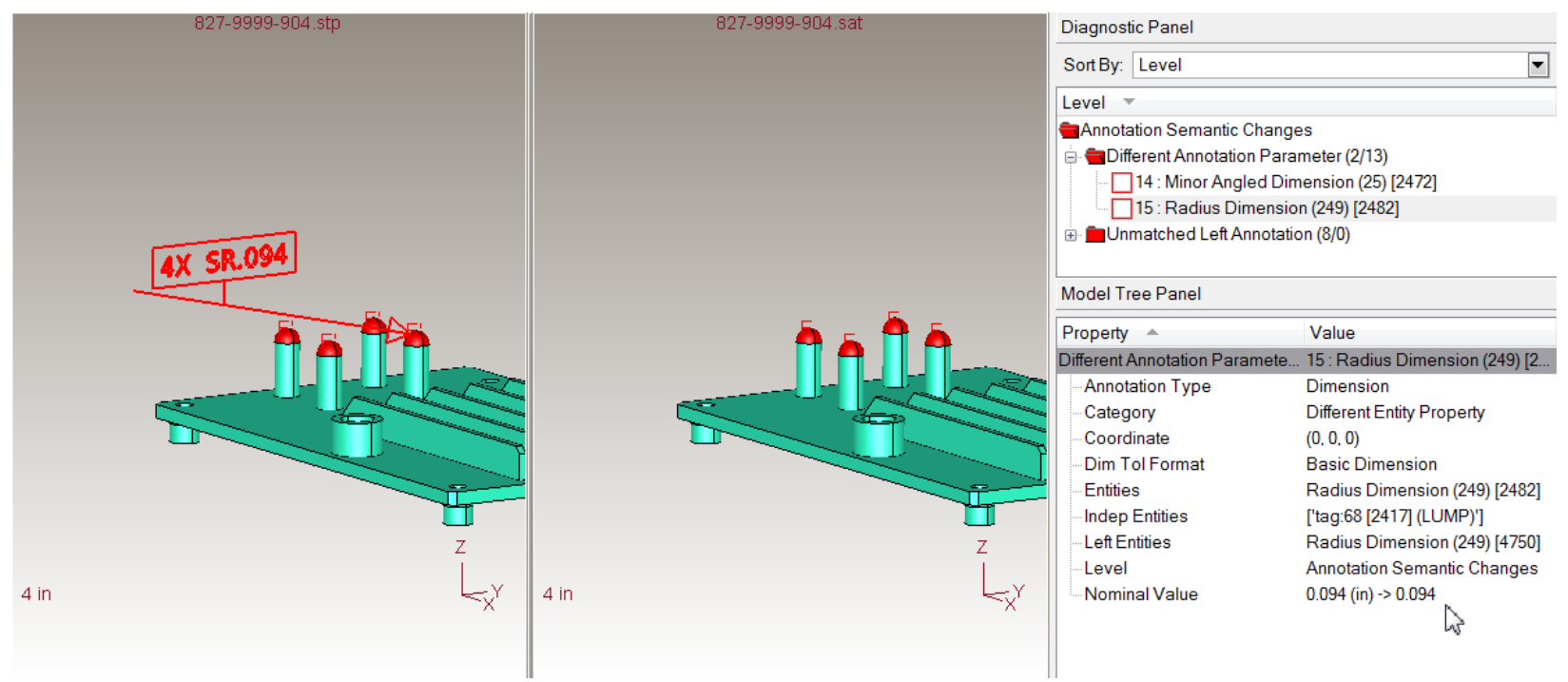

Figure 5 - Example of Validation of extended-ACIS PMI with Source STEP Model

Note that, in the example shown, though the value of the dimension was preserved during transformation, the units of the dimension value were lost. Investigation of this observed behavior suggests that adjustment to ACIS handling of units is required.

\section{Results from Validation of Test Models from NIST Data Sets}

A broader suite of models (the NIST CTCs and FTCs from Reference C and/or D were also processed to identify what additional coverage gaps might exist. Validation of the STEP data relative to the original master CAD models was published in the above references and will not be repeated here. Instead, the validation results described in this report are for the validation of the extended ACIS models as transformed from the STEP models. It is also important to note that the STEP files used in this study are models from the very latest PDES/ProSTEP CAx testing rounds. The results are cataloged in Appendix B.

An example of validation results is shown in Figure 6 (for PMI representation - see "model tree panel" lower left [STEP] and lower right [ACIS]). 


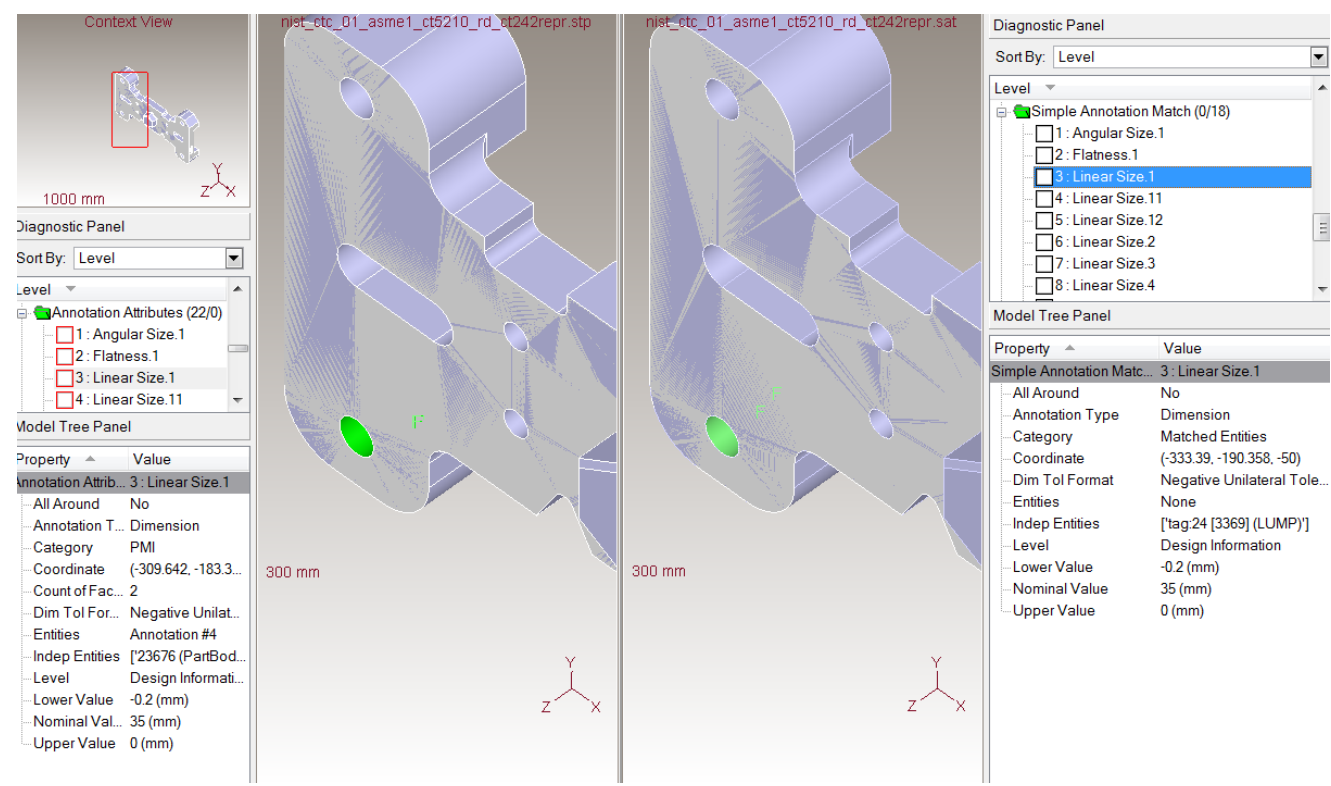

Figure 6 - Example of Successful Validation of extended-ACIS PMI representation with Source STEP Model

In this NIST CTC01 example, all semantic representation data for a linear dimension of size, including association to geometry, was successfully preserved during transformation from STEP to extended ACIS.

In a second example of validation results, shown in Figure 7 (for PMI representation - see "model tree panel" lower left [STEP] and lower right [ACIS]), the validation algorithm flags a semantic representation change due to the fact that there is no ACIS data structure for the nominal value dimension without tolerances found in the source STEP data. The current approach to work around this issue is to populate the corresponding ACIS data structure for a regular dimension with tolerances and leave the tolerance values set to zero values. 


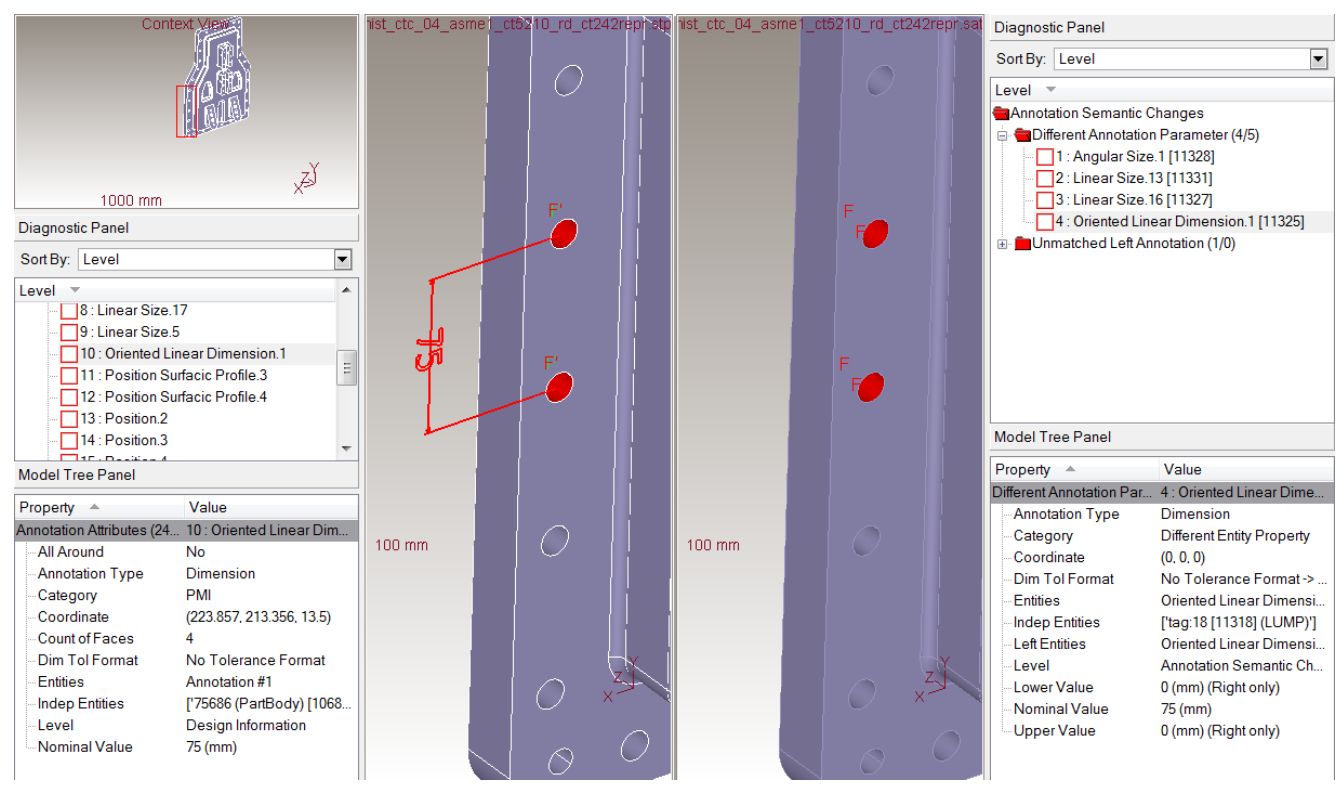

Figure 7 - Example of validation of extended-ACIS PMI representation with source STEP Model illustrating an anomaly

Additional discussion of validation results, anomalies, and likely causes will be discussed below.

\section{DISCUSSION AND CONCLUSIONS}

\section{STEP - QIF PMI Mappings}

As discussed earlier in this report, mapping between STEP and QIF resulted in some disconnects as shown in Figure 8. Some of these differences are discussed below.

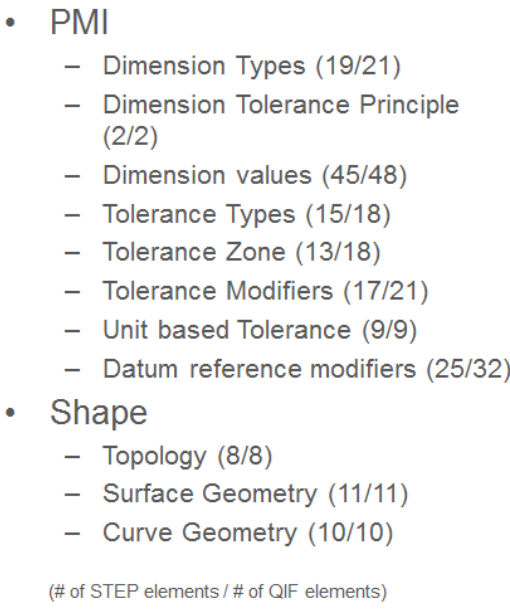

Figure 8 - Tally of mappings between STEP and QIF

There was generally good agreement between QIF and STEP for boundary representation shape and geometry. Two element types - mesh face and mesh loop - were supported in STEP and QIF but were absent in ACIS. 
The STEP-QIF working group discussed the concept of feature at length but was not able to fully close on agreement on a reasonable mapping between the two standards. The concept of "feature" is used in many systems and standards but there is often significant variation in the meaning and usage of the term among them. STEP has the concept of feature in AP242 (design feature) as well as AP238 (manufacturing feature), both represent a shape element that is flexibly defined by parameters which control the size or location of the shape element. QIF also has the concept of feature but that did not have direct STEP equivalent (26 QIF elements). It appeared to be a categorized shape aspect. The working group thinks these QIF features would map back and forth as shape aspects however they agreed to table the attempt to map features and recommend a separate research activity just to explore this construct.

Another area that should be studied further is that, in QIF, there are a number of user-defined tolerance entity types that were included in QIF but not included in STEP or ACIS. The working group agreed to table the attempt to map these UDTs and recommend follow-on research to explore this construct.

As shown in Figure 8, a number of ancillary MBD-related elements, also shown in Appendix A.5, are important for proper MBD usage and either do not currently have support in one or more standards. Examples of this include Notes, which are not supported in ACIS, and both Flag Notes and Surface Finish, which are not supported in STEP. In addition, there were two MBDrelated elements that were not supported in any of the examined standards (e.g. Tables and Global or General Tolerances). Of the two of these, the ability to capture and map global or general tolerances was considered to be a significant impediment to MBD exchange due to its widespread use in industry.

A final observation is that, in many cases, PMI constructs could be mapped for STEP and for QIF but not for ACIS. This suggests that STEP to QIF may be a better exchange mechanism than STEP to ACIS once downstream consuming systems have developedand begun delivering QIF import capabilities.

\section{Validation of Extended ACIS}

There were a significant number of anomalies in the results for validation of the extended ACIS when performed by rigorous automated means. As mentioned above, note entities were one category of PMI that was not transferred to ACIS in all test cases. This was due to the lack of an appropriate data structure to hold them in ACIS.

It was also observed that models containing Datum Target Symbols (DTS, CTCs 02 and 05) were also not well handled during the data transfer to ACIS regardless of system. Reviewing the results in Appendices B1 and B2, the overall percent success of validation, including the note and DTS anomalies, was 59\%. If the impact of these two issues is ignored, then the overall percent success improves to $67 \%$. It is worthwhile to note that in no exchange was the DTS type successfully exchanged, i.e. that all semantic structure information was included and the values of that semantic data were correct. The reasons for DTS failure were different. Three examples that illustrate those different failure modes are shown in Figures 9a, 9b, and 9c below for test case CTC02. 

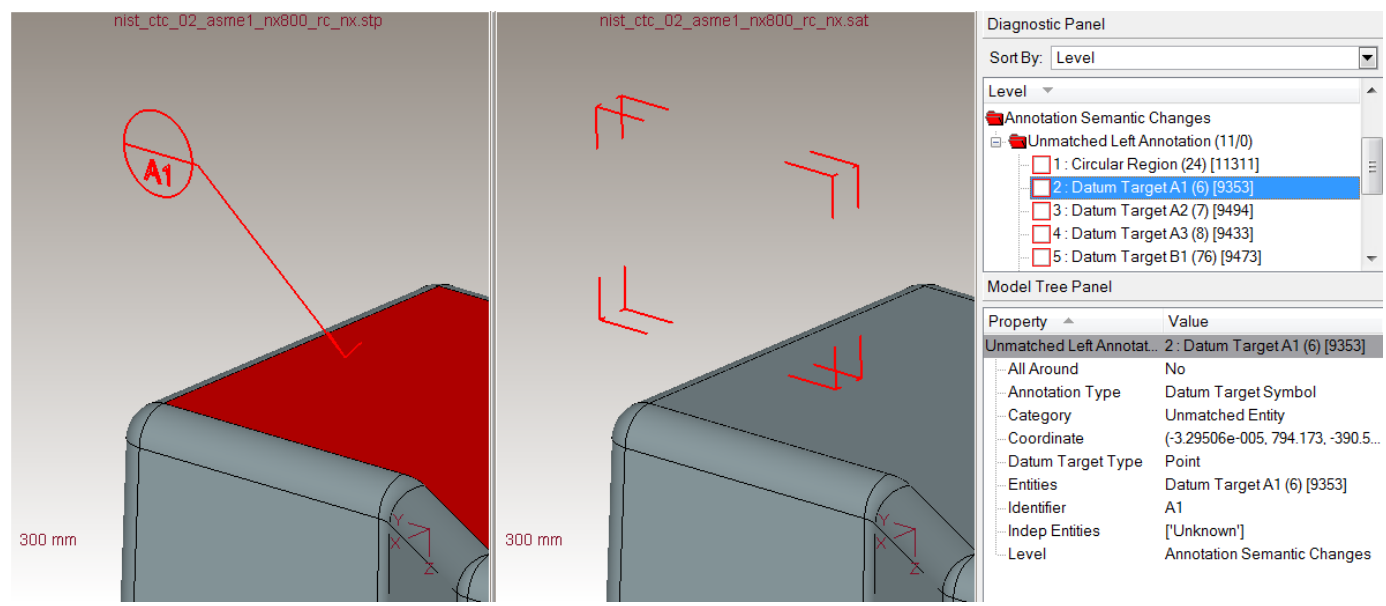

Figure 9a - Example of validation of extended-ACIS PMI representation with source STEP Model illustrating complete loss of Datum Target Symbol in the ACIS target model

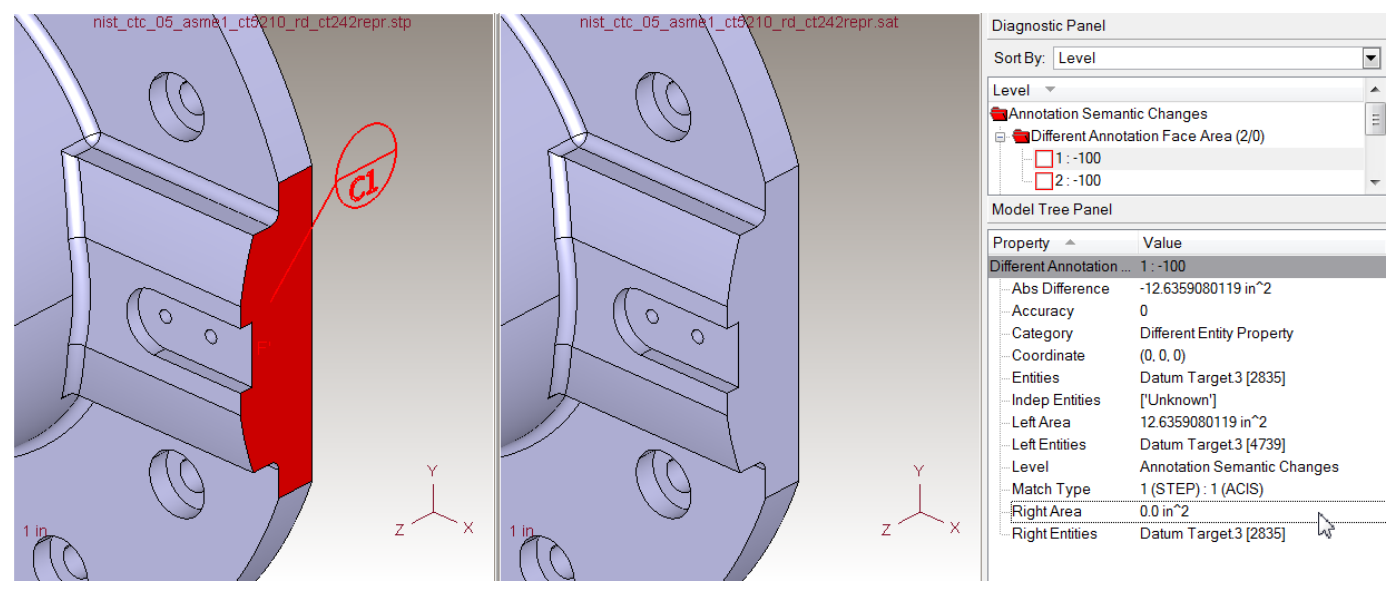

Figure 9b - Example of validation of extended-ACIS PMI representation with source STEP Model illustrating loss of Associated Geometry for a Datum Target Symbol in the target ACIS model

As shown in Figure 9a, the PMI data structure for a DTS in this particular source STEP data could not be matched to any equivalent data structure in resulting model transformed into ACIS. In a second example (Figure $9 \mathrm{~b}$ ), though the PMI data structure in the source STEP data could be matched to its equivalent data structure in resulting model transformed into ACIS, the association to the geometric surface referenced in the DTS structure was lost. Finally, in Figure 9c, the PMI data structure in the source STEP data was transferred to the resulting model in ACIS however the identifier for the DTS was changed during the exchange process. Issues with Datum Target Symbols for test case CTC05 were similar to those found in CTC02. 


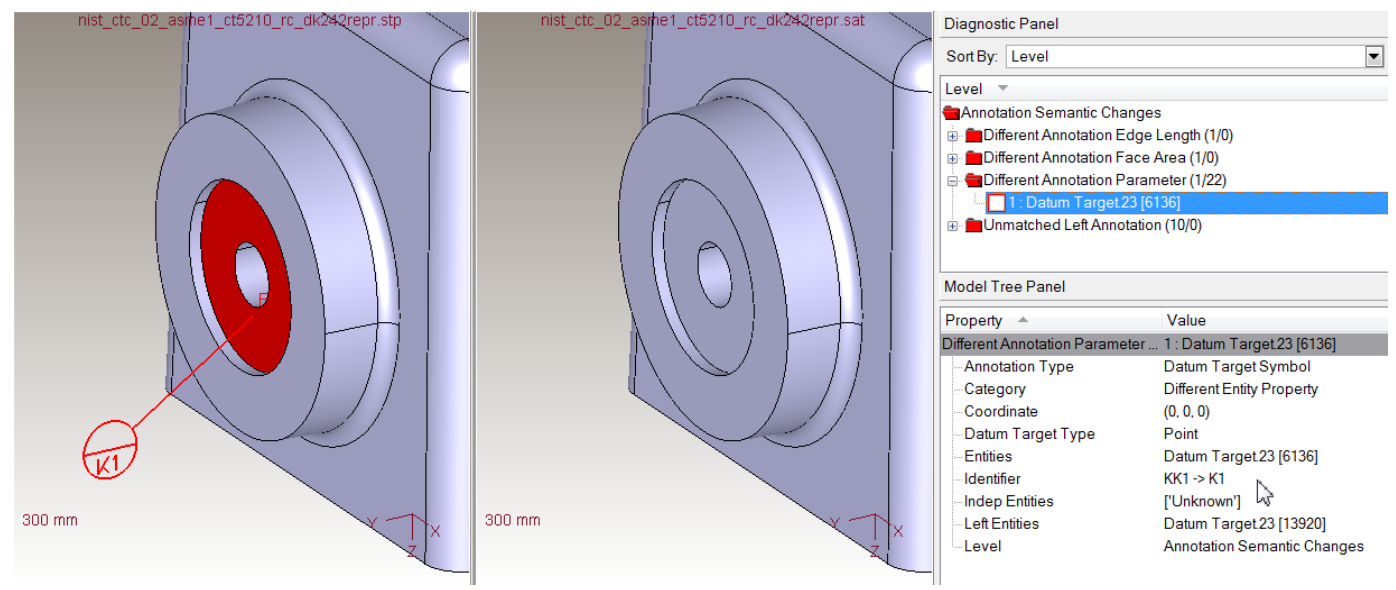

Figure 9c - Example of validation of extended-ACIS PMI representation with source STEP model illustrating an identifier change for a Datum Target Symbol in the target ACIS model

Though non-existent Note capability and widespread Datum Target Symbol failures predominated, anomalies did occur in the remaining categories. Referring to Table 4 (below), the percentage success for Datum Feature Symbols (DFS), Dimensions (DIM), and Feature Control Frames (FCF) were $72 \%, 64 \%$, and $62 \%$ respectively when Notes and DTS failures were ignored.

The results for the CTC01 test case showed the most issues, and variation between systems, in dimensions. Datum Feature Symbols and Feature Control Frames were generally stable across systems with close correlation between systems. The results for CTC3 and CTC4 test case were reasonably good for all vendors, averaging $93 \%$ and $84 \%$ success, respectively, when note failures were ignored. Dimensions were, again, the type with the most issues.

As stated earlier, the results for the CTC05 test case showed problems for Datum Target Symbols (DTS). If DTS errors and note failures are ignored, the results for CTC05 averaged $62 \%$ success. 
Table 4: Validation of NIST models using analysis software

\begin{tabular}{|c|c|c|c|c|c|}
\hline Model File & $\begin{array}{l}\text { DFS }^{4} \\
\text { Clean }\end{array}$ & $\begin{array}{l}\text { DIM }^{5} \\
\text { Clean }\end{array}$ & $\begin{array}{l}\mathrm{FCF}^{6} \\
\text { Clean }\end{array}$ & $\begin{array}{c}\text { Percent }^{\top} \\
\text { Clean } \\
(\mathrm{xN})\end{array}$ & $\begin{array}{c}\text { Percent }^{8} \\
\text { Clean } \\
\text { (xNDTS) }\end{array}$ \\
\hline nist_ctc_01_asme1_ct5210_rd_ct242repr.stp & 2 & 7 & 6 & $79 \%$ & $79 \%$ \\
\hline nist_ctc_01_asme1_ct5210_rd_dk242repr.stp & 3 & 6 & 6 & $79 \%$ & $79 \%$ \\
\hline nist_ctc_01_asme1_nx800_rd_ct242repr.stp & 3 & 7 & 6 & $84 \%$ & $84 \%$ \\
\hline nist_ctc_01_asme1_nx800_rd_nx.stp & 3 & 1 & 6 & $53 \%$ & $53 \%$ \\
\hline nist_ctc_01_asme1_nx800_rd_th.stp & 3 & 9 & 6 & $95 \%$ & $95 \%$ \\
\hline nist_ctc_02_asme1_ct5210_rc_ct242repr.stp & 0 & 0 & 0 & $0 \%$ & $0 \%$ \\
\hline nist_ctc_02_asme1_ct5210_rc_dk242repr.stp & 0 & 0 & 0 & $0 \%$ & $0 \%$ \\
\hline nist_ctc_02_asme1 nx800 rc_ct242repr.stp & 0 & 0 & 0 & $0 \%$ & $0 \%$ \\
\hline nist_ctc 02 asme 1 nx 800 rc nx.stp & 6 & 7 & 22 & $80 \%$ & $100 \%$ \\
\hline nist_ctc 02 asme $1 \mathrm{nx} 800$ rc th.stp & 0 & 0 & 0 & $0 \%$ & $0 \%$ \\
\hline nist_ctc_03 asme1_ct5210_rc_ct242repr.stp & 6 & 8 & 13 & $93 \%$ & $93 \%$ \\
\hline nist_ctc_03_asme1_ct5210_rc_dk242repr.stp & 6 & 8 & 13 & $93 \%$ & $93 \%$ \\
\hline nist_ctc_03_asme1_nx800_rc_ct242repr.stp & 6 & 8 & 13 & $93 \%$ & $93 \%$ \\
\hline nist_ctc_03_asme1_nx800_rc_nx.stp & 6 & 8 & 13 & $93 \%$ & $93 \%$ \\
\hline nist_ctc_03 asme1_nx800_rc th.stp & 6 & 9 & 13 & $97 \%$ & $97 \%$ \\
\hline nist_ctc_04_asme1_ct5210_rd_ct242repr.stp & 8 & 7 & 5 & $87 \%$ & $87 \%$ \\
\hline nist_ctc_04_asme1_ct5210_rd_dk242repr.stp & 8 & 9 & 3 & $83 \%$ & $83 \%$ \\
\hline nist_ctc_04_asme1_nx800_rd_ct242repr.stp & 8 & 7 & 4 & $83 \%$ & $83 \%$ \\
\hline nist_ctc_04_asme1_nx800_rd_nx.stp & 8 & 5 & 5 & $78 \%$ & $78 \%$ \\
\hline nist_ctc_04_asme1_nx800_rd_th.stp & 8 & 7 & 5 & $87 \%$ & $87 \%$ \\
\hline nist_ctc_05 asme1_ct5210 rd_ct242repr.stp & 4 & 2 & 6 & $55 \%$ & $60 \%$ \\
\hline nist_ctc_05_asme1_ct5210_rd_dk242repr.stp & 4 & 3 & 7 & $64 \%$ & $70 \%$ \\
\hline nist_ctc_05_asme1_nx800_rd_ct242repr.stp & 2 & 2 & 5 & $45 \%$ & $50 \%$ \\
\hline nist_ctc_05_asme1_nx800_rd_nx.stp & 2 & 2 & 10 & $70 \%$ & $78 \%$ \\
\hline nist_ctc_05 asme1 nx800 rd th.stp & 2 & 2 & 6 & $45 \%$ & $50 \%$ \\
\hline Counts: & 107 & 178 & 186 & $62 \%$ & $67 \%$ \\
\hline Percents: & $72 \%$ & $64 \%$ & $62 \%$ & & \\
\hline
\end{tabular}

Error conditions for Feature Control Frame included loss of geometric associativity, either loss or changes to primary datum reference frame identifiers, or loss of FCF type. Several examples of these losses are shown in Figures 10a through 10d.

${ }^{4}$ DFS $=$ Datum Feature Symbol

${ }^{5} \mathrm{DIM}=$ Dimension

${ }^{6} \mathrm{FCF}=$ Feature Control Frame

${ }^{7} \mathrm{xN}=$ Percent Clean excluding Note entities

${ }^{8}$ xNDTS $=$ Percent Clean excluding Note and Datum Target Symbols 

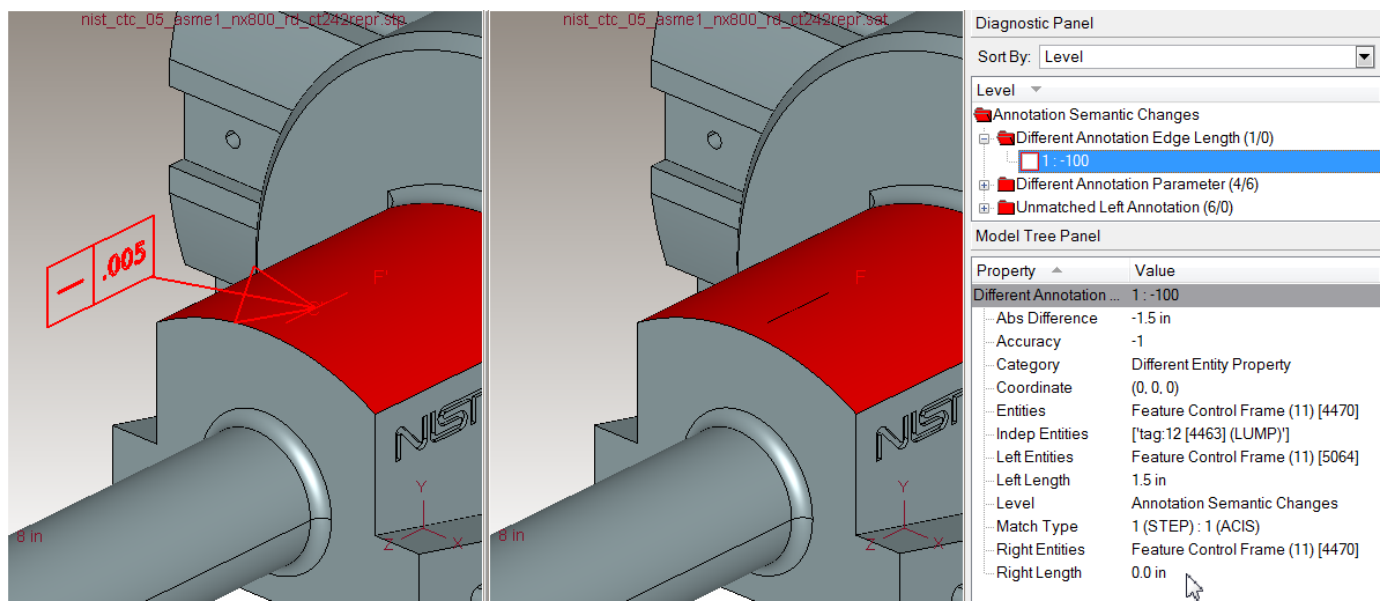

Figure 10a - Loss of Feature Control Frame associativity to associated edge geometry
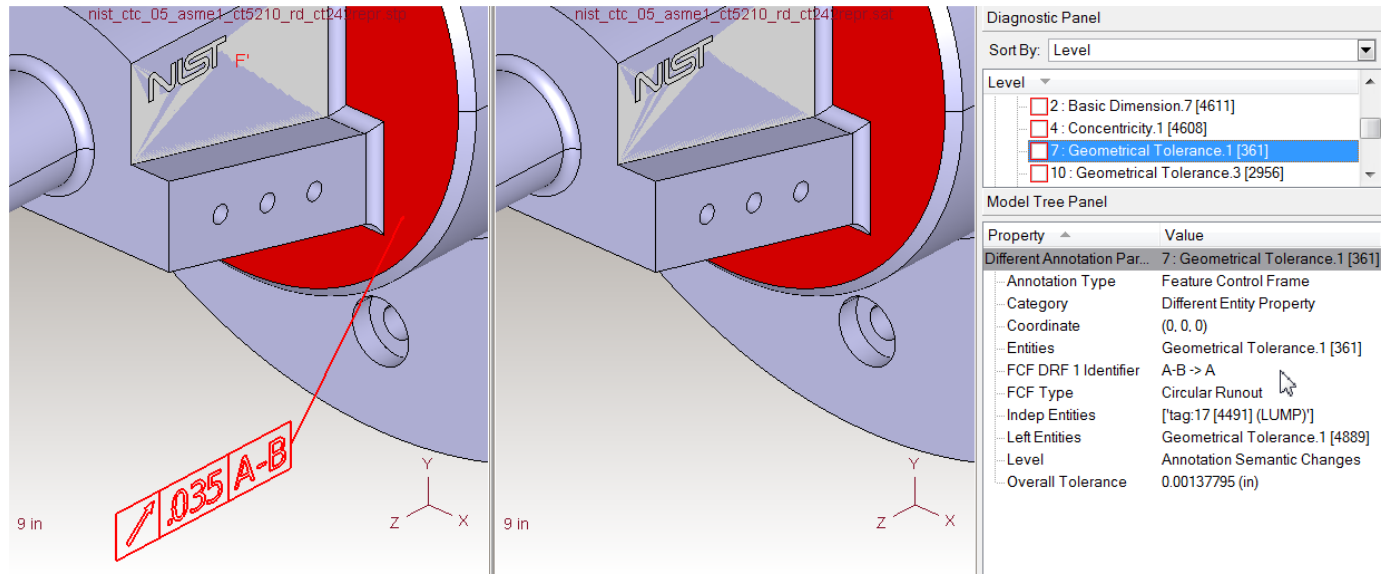

Figure 10b - Change to Feature Control Frame primary datum reference frame identifier

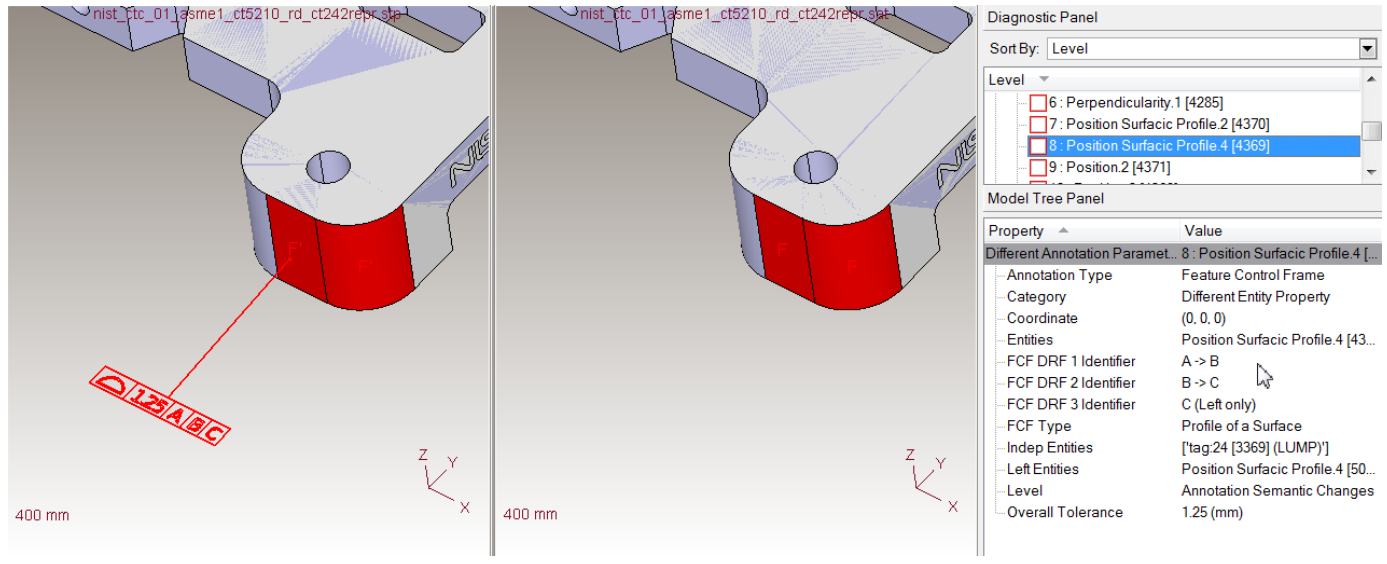

Figure 10c - Identifier of Feature Control Frame primary datum reference frame not translated 

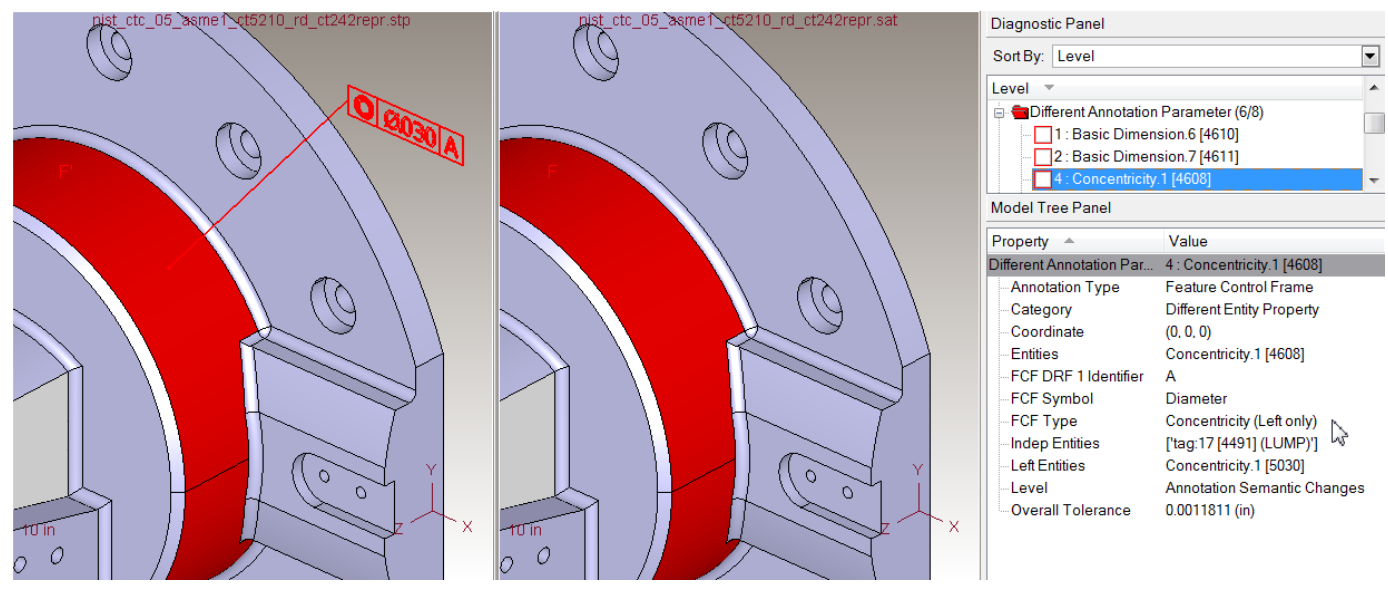

Figure 10d - Loss of Feature Control Frame type

Dimension-related errors are illustrated in Figures 11a through 11h below. In Figure 11a below, the error condition indicates that the values for the nominal and tolerance values have changed. A quick calculation shows that the difference is related to improper conversion as the values on the left differ to those on right by a factor equal to the conversion factor from degrees to radians. It is unclear without further investigation whether the effort occurs in the translation from STEP to ACIS or in the validation algorithm display.

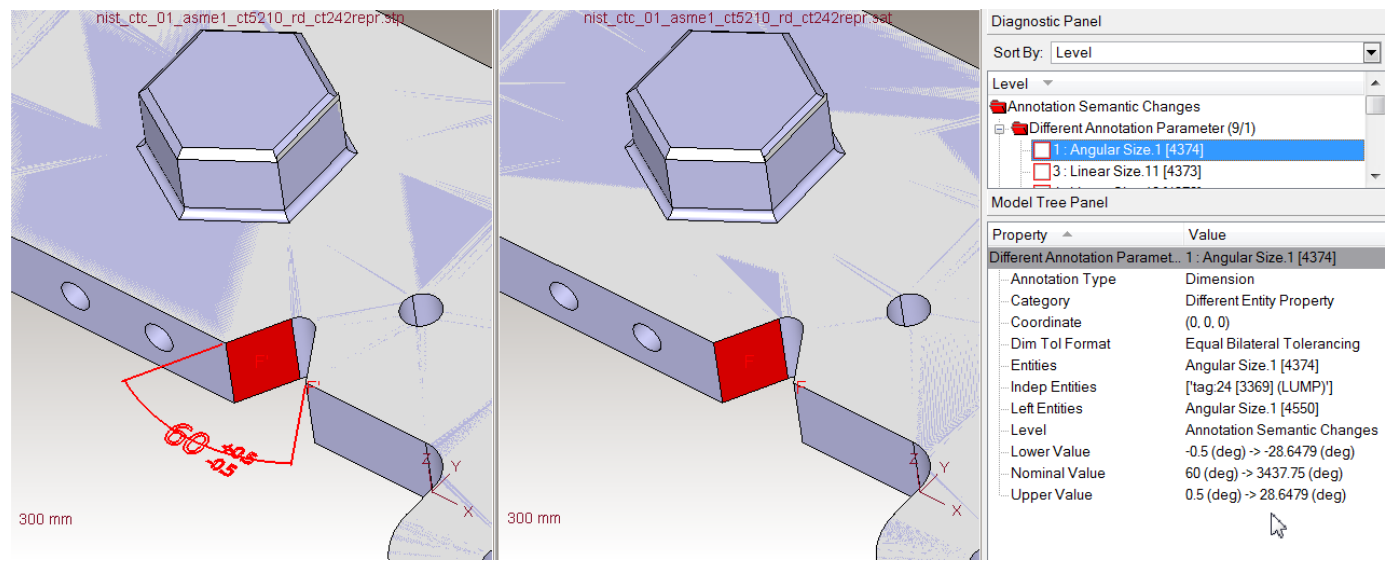

Figure 11a - Angular bilateral dimension nominal and tolerance values changed

Figure 11b illustrates a problem with extended ACIS in that there is no separate data structure for limit dimensions. To provide a workaround, dimensions of this type were recast as nominal dimensions with upper and lower tolerances when converted from STEP to ACIS. The validation algorithm needs to be adjusted to understand this workaround. Figure 11c shows the loss of units on tolerance values. Representation of the proper units in the ACIS data is critical to reuse in the Metrology planning system. The use of not-to-scale dimensions, in Figure 11d, 

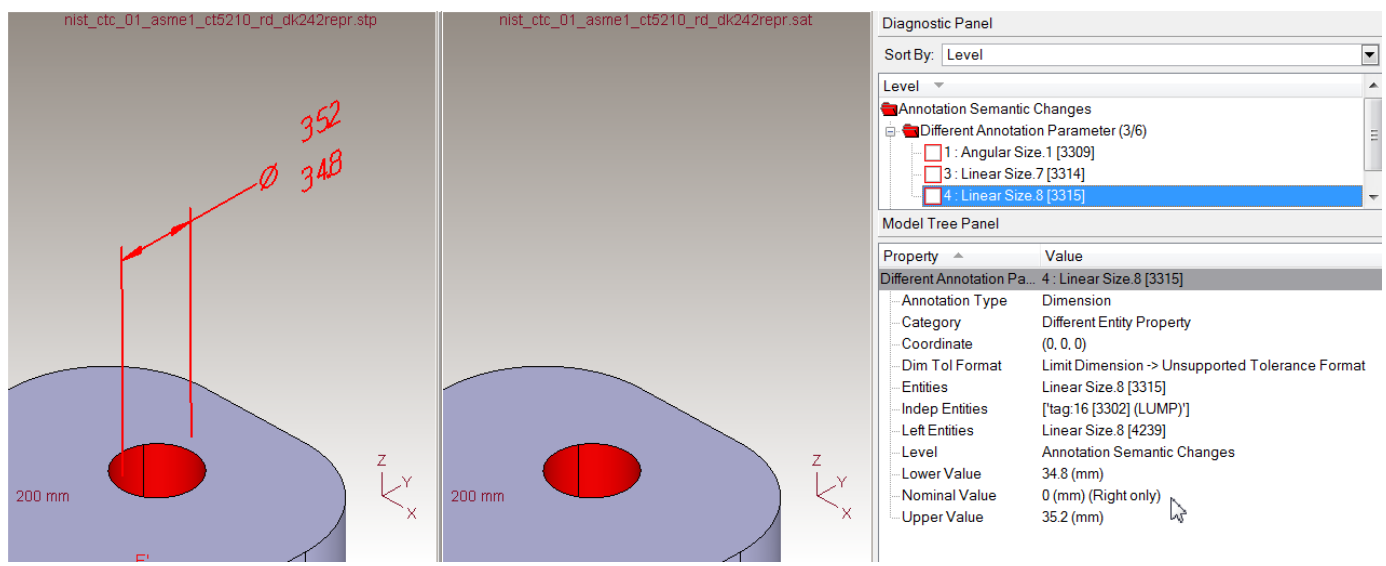

Figure 11b - Limit dimension nominal value added and set to zero
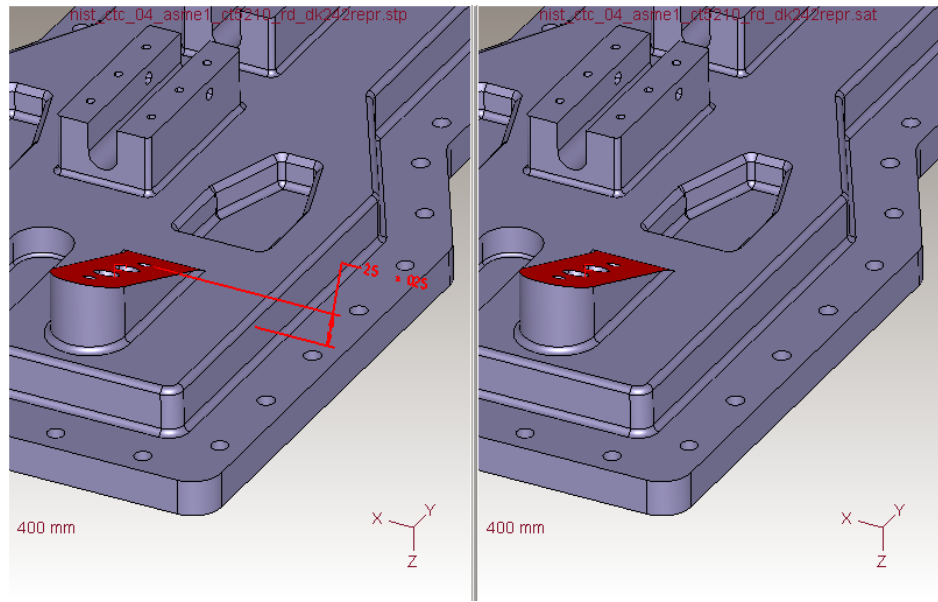

\begin{tabular}{|c|c|}
\hline \multicolumn{2}{|l|}{ Diagnostic Panel } \\
\hline \multicolumn{2}{|l|}{ Sort By: Level } \\
\hline \multicolumn{2}{|l|}{ Level $\mathrm{r}$} \\
\hline \multirow{2}{*}{\multicolumn{2}{|c|}{$\begin{array}{l}\text { Annotation Semantic Changes } \\
\text { Different Annotation Parameter (2/4) }\end{array}$}} \\
\hline & \\
\hline \multicolumn{2}{|c|}{$\square$ 1: Angular Size.1 [10091] } \\
\hline \multicolumn{2}{|c|}{ 2: Linear Size 16 [10096] } \\
\hline \multicolumn{2}{|c|}{ Unmatched Left Annotation (4/0) } \\
\hline \multicolumn{2}{|l|}{ Model Tree Panel } \\
\hline 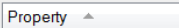 & Value \\
\hline Different Annotation Par... & 2: Linear Size.16 [10096] \\
\hline Annotation Type & Dimension \\
\hline Category & Different Entity Property \\
\hline Coordinate & $(0,0.0)$ \\
\hline Dim Tol Format & Equal Bilateral Tolerancing \\
\hline Entities & Linear Size. 16 [10096] \\
\hline -Indep Entities & ['tag:18 [10067] (LUMP)'] \\
\hline Leff Entities & Linear Size. $16[11358]$ \\
\hline Level & Annotation Semantic Changes \\
\hline Lower Value & $-0.25(\mathrm{~mm}) \rightarrow-0.25$ \\
\hline Nominal Value & $25(\mathrm{~mm}) \rightarrow 25.0$ \\
\hline Upper Value & $0.25(\mathrm{~mm})>0.25$ \\
\hline
\end{tabular}

Figure 11c - Units on linear bilateral dimension nominal and tolerance values lost
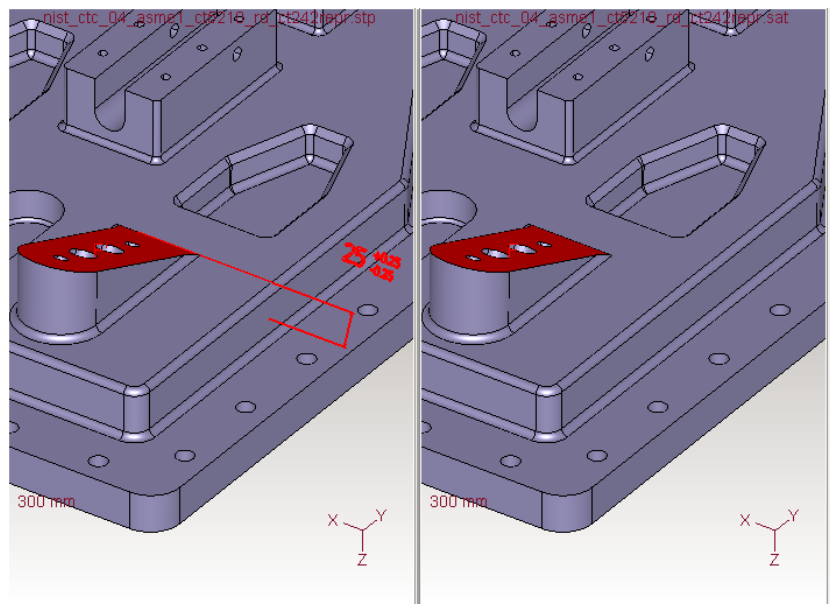

\begin{tabular}{|c|c|c|}
\hline \multicolumn{2}{|l|}{ Panel } & \\
\hline \multicolumn{3}{|l|}{ Sort By: Level } \\
\hline \multicolumn{2}{|l|}{ Level $\mathrm{F}$} & - \\
\hline \multirow{2}{*}{\multicolumn{3}{|c|}{$\begin{array}{l}\text { Annotation Semantic Changes } \\
\text { Different Annotation Parameter (4/5) }\end{array}$}} \\
\hline & arameter (4/5) & \\
\hline \multicolumn{3}{|c|}{$\square$ 1: Angular Size. 1 [11328] } \\
\hline \multicolumn{3}{|c|}{$\square$ 2: Linear Size. 13 [11331] } \\
\hline \multicolumn{3}{|c|}{$\square$ 3: Linear Size. 16 [11327] } \\
\hline \multicolumn{3}{|c|}{$\square$ 4: Oriented Linear Dimension. 1 [11325] } \\
\hline \multicolumn{3}{|l|}{ Model Tree Panel } \\
\hline Property $\triangle$ & Value & \\
\hline Different Annotation Param... & 3: Linear Size. 16 [11327] & \\
\hline Annotation Type & Dimension & \\
\hline Category & Different Entity Property & \\
\hline Coordinate & $(0,0,0)$ & \\
\hline Dim Tol Format & Not To Scale $>>$ Unsupported Tolerance Forma & \\
\hline Entities & Linear Size.16 [11327] & \\
\hline -Indep Entities & ['tag:18 [11318] (LUMP)'] & \\
\hline Left Entities & Linear Size. 16 [10735] & \\
\hline Level & Annotation Semantic Changes & \\
\hline Lower Value & $-0.2(\mathrm{~mm})$ (Right only) & \\
\hline Nominal Value & 20 (mm) (Right only) & \\
\hline Upper Value & $0.2(\mathrm{~mm})$ (Right only) & \\
\hline
\end{tabular}

Figure 11d - Not to scale dimension nominal and tolerance values uncertain 
shows a particularly challenging problem for automated systems which will need to account for such uses in 3D data. This connection between a native dimension's to-scale value and its notto-scale value were likely lost during conversion. Further investigation is required.

In the example of Figure 11e below, a non-toleranced reference dimension has the same issue as previously discussed on page 21 for limit dimensions. The validation algorithm needs to be adjusted to understand the recasting of non-toleranced and limit dimension workaround.

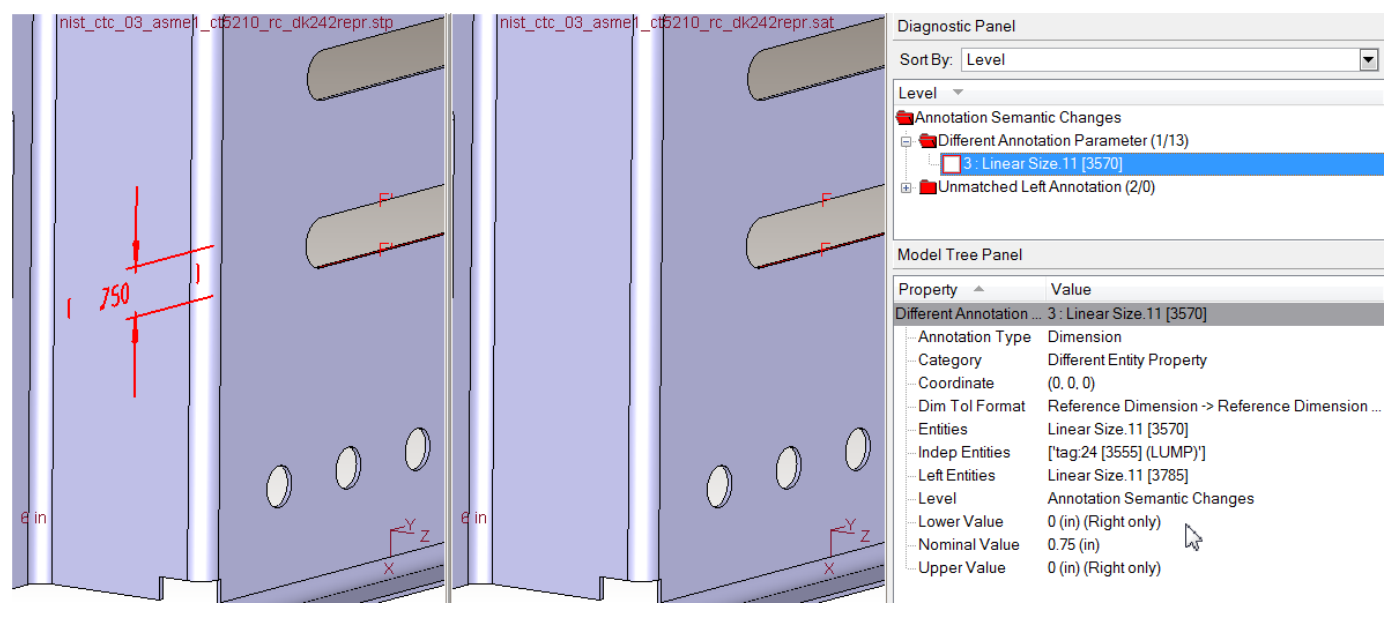

Figure 11e - Reference dimension with no tolerances gets tolerances set to zero

The final three error types will require some more detailed analysis. Each of these has been analyzed by the validation algorithm as being "unformated dimensions". This suggests that there were issues when attempting to read the STEP entity and further analysis of the entire conversion process is necessary to understand exactly where the anomaly occurred.
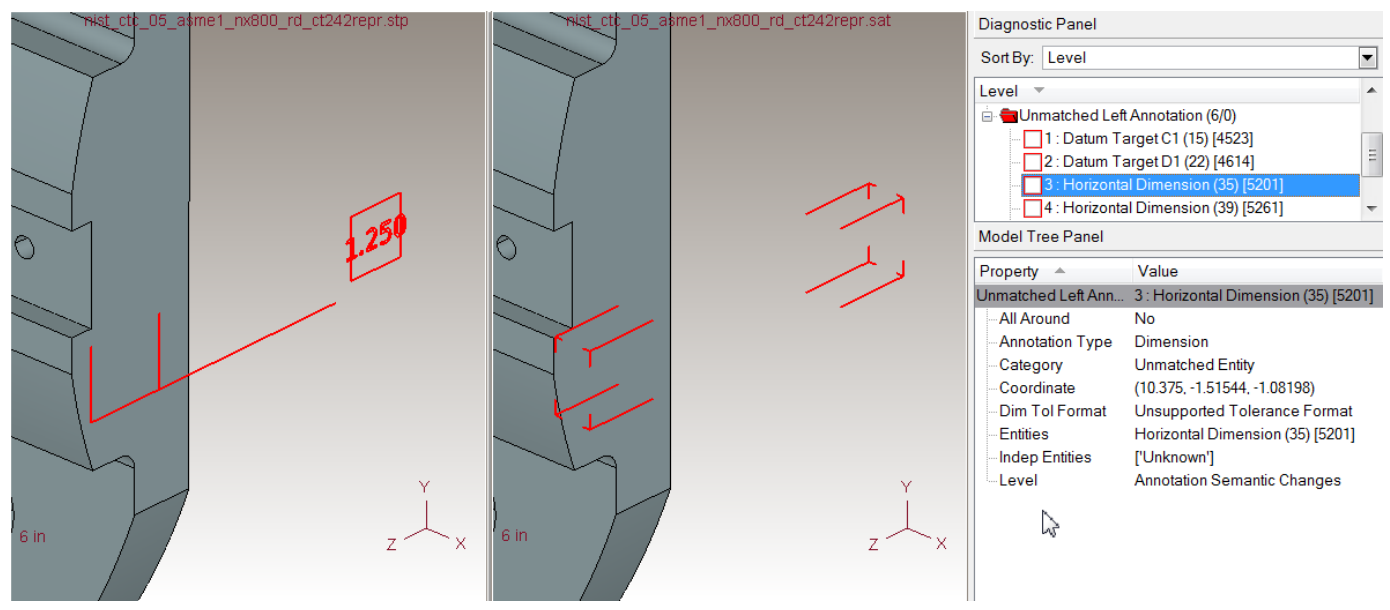

Figure 11f - Unformatted dimension with no nominal value not translated 


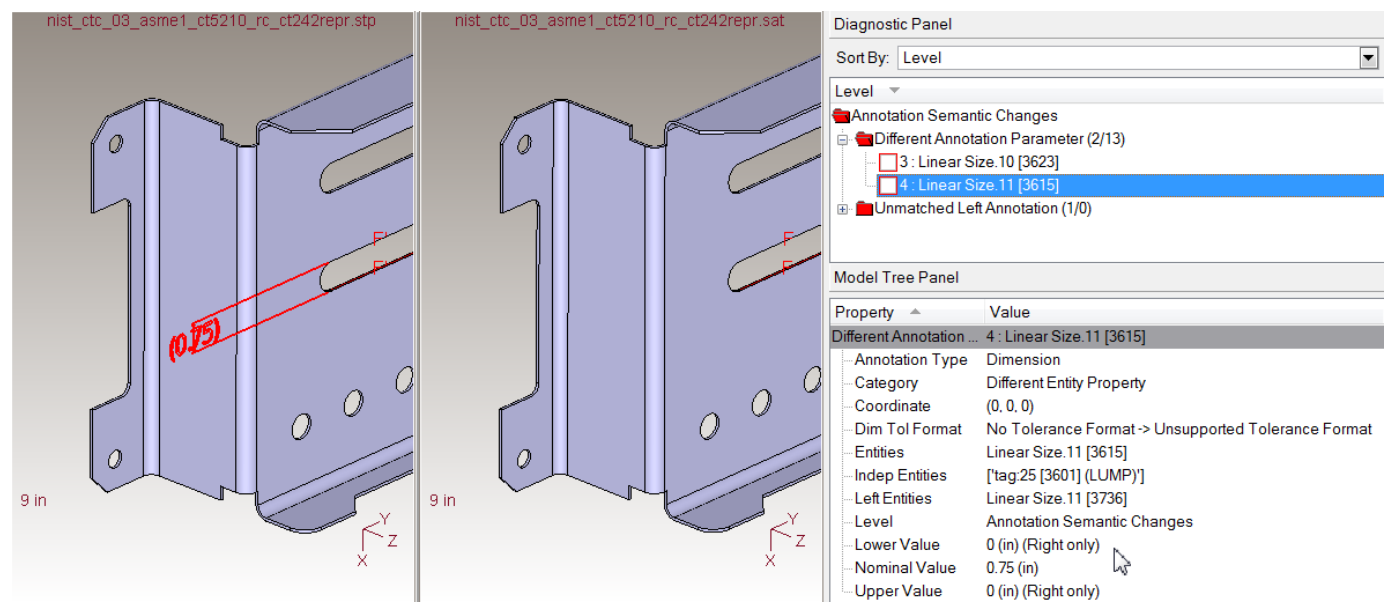

Figure 11g - Unformatted dimension with no tolerances gets tolerances set to zero

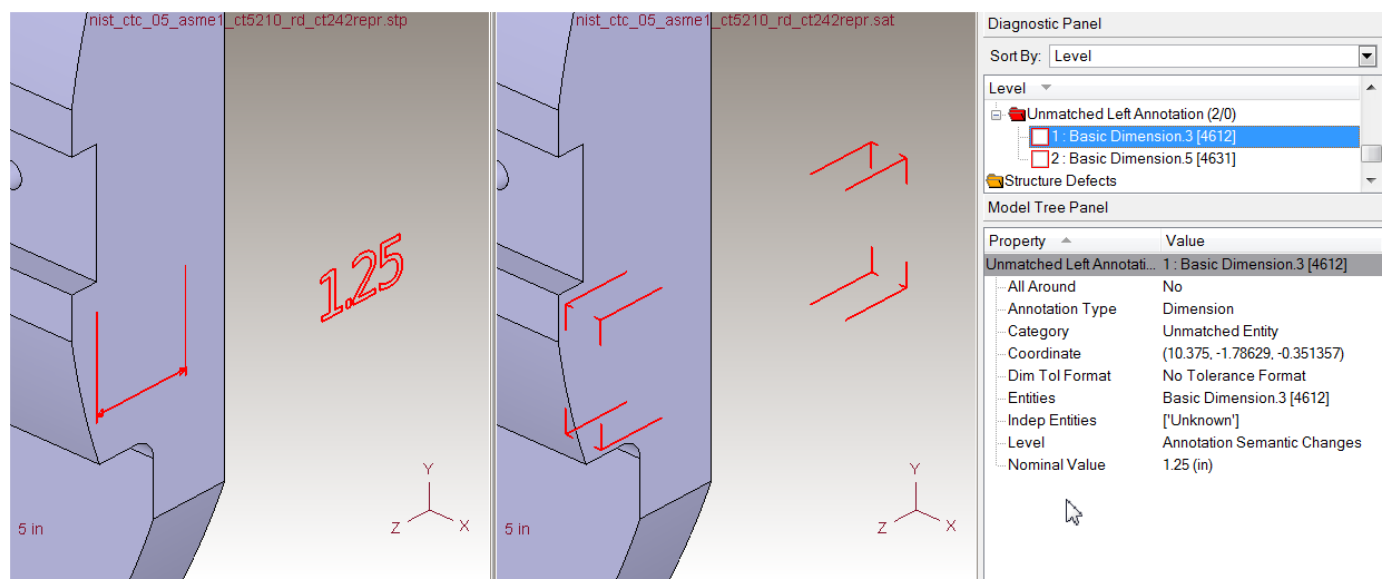

Figure $11 \mathrm{~h}$ - Unformatted dimension with no tolerances not translated

Though some issues with PMI mapping into extended ACIS have been resolved and coverage for many typical PMI constructs are supported, other PMI mappings into extended ACIS remain unresolved due to problems in ACIS. Those unresolved ACIS issues will likely remain open issues for consideration as further research in a future project.

\section{Conclusions}

This project sought to provide initial research necessary to understand the unique nature of CAM and CM community's needs in the area of interoperability between upstream design systems and those CAM and CM systems and how their needs map to existing standards, the development of algorithms to perform these validations of CAM and CM models, and testing these validation processes on a representative set of example CAD models that have been read by CAM and CM systems, validating these CAM and CM data models against their STEP source models for data loss during transformation. 
ACIS is a typical modeling kernel in the CAM and CM space and would seem to be a good target for a transformation and validation process for passing design data downstream to these systems. The research hypothesized here suggested that using a STEP AP242 to extended ACIS translation process and following that translation with an ACIS to STEP validation would be good first step toward the promise of true MBD, i.e. the ability to share design data including PMI with downstream consumers in the manufacturing and metrology spaces. The results of this research show that there is a clear requirement for validation, as transformation of semantic data structures needed for machine consumption downstream require in-depth automated validation processes to ensure data integrity, to establish confidence in the transformed data, and to flag any losses during the transformation process.

A further conclusion, based on this research is that it is possible to use ACIS as the target for conversion of data from STEP AP242. There are, however, gaps in the process and recommended practices and algorithm refinements are required to resolve roadblocks and fully realize the potential value of MBD data in the CAM and CM domains. The mapping exercise performed between STEP AP242, ACIS, and the Metrology community's Quality Information Framework (QIF) standard suggests that transformation between STEP and QIF might be a strong contender as the mechanism of choice for exchange between design and metrology. The mappings identify data model elements, properties, and relationships which are unique to each standard and those which are equivalent between them. QIF has unique capabilities, not shared with STEP AP242, but which are necessary to properly communicate metrology data and process.

This study benefits end users, software developers, and standards experts by helping all of these groups better understand the relative strengths and weaknesses of each standard. It will also assist developers to improve translation and validation applications between the standards. And it will provide strategic direction for the standards experts as they seek to refine and further harmonize the standards, improving interoperability between the design domain and the manufacturing and metrology domains over time.

\section{ACKNOWLEDGEMENTS}

The team would first like to recognize the NIST System Integration Division, Engineering Laboratory, for providing this project opportunity. The Digital Thread is an important concept for industry. NIST focus and investment in product lifecycle capabilities and standards is truly recognized. Especially, we thank Allison Barnard Feeney and Tom Hedberg who have provided the leadership in establishing the Digital Thread for Smart Manufacturing. The project team fully supports the drive to investigate the product lifecycle holistically and extend the digital thread of information with easy implementation into manufacturing systems. NIST has provided a significant seed opportunity for commercialization of standards-based model-centric data interoperability - enabling industry's vision of the Model-Based Enterprise.

A project like this would not be possible without the commitment and contributions from a number people. The project team would like to acknowledge the following companies and individuals who participated in various elements of the grant. 
Honeywell FMT, Kansas City, MO, provided consulting services for the STEP- QIF mapping activity. Curtis Brown of Honeywell FMT contributed to an understanding of the current and expected near-term states of the QIF standard. Curtis also contributed significantly to development of the STEP - QIF mapping.

Capvidia, Leuven, Belgium, provided consulting services for the STEP- QIF mapping activity. Tomazs Luniewski and Victor Mikushin of Capvida contributed to an understanding of the structure of the QIF standard and how the QIF standard is implemented in practice. Victor also contributed significantly to development of the STEP - QIF mapping.

Daniel Campbell, of Metrosage and Tom Kramer and Hui-Min Huang of NIST provided valuable editorial contribution to the STEP- QIF mapping activity.

International TechneGroup Incorporated, Milford, $\mathrm{OH}$, provided overall program execution, grant administration, and project management as well as technical development of validation components and validation analysis of model data. Ed Paff was responsible for translation component development as well as providing insights into the current and expected near-term states of the ISO10303 STEP standard relating to PMI, as well as development of the PMI mapping framework and STEP mapping into the framework. Dave Smith was responsible for technical development of validation algorithms. Doug Cheney was responsible for validation analyses of model data. Asa Trainer was responsible for program execution, administration, and project management. Asa contributed significantly to authoring of the final report. 


\section{REFERENCES *}

\begin{tabular}{|l|l|}
\hline Reference & Description \\
\hline Reference A & $\begin{array}{l}\text { NIST 70NANB14H314 - Collaborative Agreement - Investigating the Impact } \\
\text { of Standards-based Interoperability for Design to Manufacturing and Quality } \\
\text { in the Supply Chain }\end{array}$ \\
\hline Reference B & $\begin{array}{l}\text { Contract Number SB1341-12-SE-0853 - NIST 3D Model-Based Engineering } \\
\text { Product Manufacturing Information Validation and Conformance Testing } \\
\text { Project - Phase 1 }\end{array}$ \\
\hline Reference C & $\begin{array}{l}\text { Contract Number SB1341-14-SE-0061 - NIST 3D Model-Based Engineering Product } \\
\text { Manufacturing Information Validation and Conformance Testing } \\
\text { Project - Phase 2 }\end{array}$ \\
\hline Reference D & ISO 10303-242 "Managed Model Based 3D Engineering" [STEP AP 242] \\
\hline Reference E & ANSI QIF 2.0, October 31, 2014 - http://qifstandards.org/download-qif/ \\
\hline
\end{tabular}




\section{APPENDICES}

\section{Appendix A: Mapping PMI into STEP and QIF}

Appendix A.1: Mapping GD\&T into STEP and QIF

\begin{tabular}{|c|c|c|c|}
\hline PMI & STEP AP242 & ACIS & $\underline{\text { QIF }}$ \\
\hline \multicolumn{4}{|l|}{ dimension types } \\
\hline linear dimension & dimensional_location & spaxpmi_dimension & $\begin{array}{l}\text { LinearCharacteristicDefinitionBas } \\
\text { eType }\end{array}$ \\
\hline radius dimension & dimensional_size.name = "radius" & spaxpmi_dimension & $\begin{array}{l}\text { RadiusCharacteristicDefinitionTy } \\
\text { pe }\end{array}$ \\
\hline spherical radius dimension & $\begin{array}{l}\text { dimensional_size } . \text { name }=\text { "spherical } \\
\text { radius" }\end{array}$ & not covered & \\
\hline diameter dimension & dimensional_size.name = "diameter" & spaxpmi_dimension & $\begin{array}{l}\text { DiameterCharacteristicDefinition } \\
\text { Type }\end{array}$ \\
\hline spherical diameter dimension & $\begin{array}{l}\text { dimensional_size.name = "spherical } \\
\text { diameter" }\end{array}$ & not covered & $\begin{array}{l}\text { SphereFeatureDefinitionType<> } \\
\text { Diameter [also in Spherical } \\
\text { Segment] }\end{array}$ \\
\hline oriented dimension & oriented_dimensional_location & not covered & $\begin{array}{l}\text { OrientationCharacteristicDefinitio } \\
\text { nBaseType }\end{array}$ \\
\hline curved dimension & $\begin{array}{l}\text { dimensional_location_with_path/dimen } \\
\text { sional_size_with_path }\end{array}$ & not covered & $\begin{array}{l}\text { CurvedLengthCharacteristicDefin } \\
\text { itionType }\end{array}$ \\
\hline length dimension & & & $\begin{array}{l}\text { LengthCharacteristicDefinitionTy } \\
\text { pe }\end{array}$ \\
\hline depth dimension & dimensional_location.name = "depth" & not covered & $\begin{array}{l}\text { DepthCharacteristicDefinitionTyp } \\
\text { e }\end{array}$ \\
\hline width dimension & dimensional_location.name = "width" & not covered & $\begin{array}{l}\text { WidthCharacteristicDefinitionTyp } \\
\text { e }\end{array}$ \\
\hline height dimension & dimensional_location.name = "height" & not covered & $\begin{array}{l}\text { HeightCharacteristicDefinitionTy } \\
\text { pe }\end{array}$ \\
\hline thickness dimension & dimension_size.name = "thickness" & not covered & $\begin{array}{l}\text { ThicknessCharacteristicDefinition } \\
\text { Type }\end{array}$ \\
\hline linear coordinate dimension & $\begin{array}{l}\text { dimensional_location.name = } \\
\text { "coordinate" }\end{array}$ & $\begin{array}{l}\text { spaxpmi_dimension(DIMSUBTYPE_CO } \\
\text { ORDDIM2D, } \\
\text { DIMSUBTYPE_COORDDIM3D) }\end{array}$ & $\begin{array}{l}\text { LinearCoordinateCharacteristicD } \\
\text { efinitionType }\end{array}$ \\
\hline angular coordinate dimension & angular_location.name = "coordinate" & $\begin{array}{l}\text { spaxpmi_dimension(DIMSUBTYPE_CO } \\
\text { ORDDIM2D, } \\
\text { DIMSUBTYPE_COORDDIM3D) }\end{array}$ & $\begin{array}{l}\text { AngularCoordinateCharacteristic } \\
\text { DefinitionType }\end{array}$ \\
\hline
\end{tabular}




\begin{tabular}{|c|c|c|c|}
\hline PMI & STEP AP242 & ACIS & QIF \\
\hline distance between dimension & $\begin{array}{l}\text { derived_shape_aspect/centre_of_sym } \\
\text { metry }\end{array}$ & not covered & $\begin{array}{l}\text { DistanceBetweenCharacteristicD } \\
\text { efinitionType }\end{array}$ \\
\hline distance from dimension & directed_dimensional_location & not covered & $\begin{array}{l}\text { DistanceFromCharacteristicDefin } \\
\text { itionType }\end{array}$ \\
\hline chord dimension & dimensional_location.name = "chord" & not covered & $\begin{array}{l}\text { ChordCharacteristicDefinitionTyp } \\
\text { e }\end{array}$ \\
\hline square dimension & & & $\begin{array}{l}\text { SquareCharacteristicDefinitionTy } \\
\text { pe }\end{array}$ \\
\hline angular dimension & angular_location/angular_size & $\begin{array}{l}\text { spaxpmi_dimension (no way to specify } \\
\text { which angle) }\end{array}$ & $\begin{array}{l}\text { AngleCharacteristicDefinitionTyp } \\
\mathrm{e}\end{array}$ \\
\hline angle-from dimension & not covered & not covered & $\begin{array}{l}\text { AngleFromCharacteristicDefinitio } \\
\text { nType }\end{array}$ \\
\hline angle-between dimension & $\begin{array}{l}\text { derived_shape_aspect/centre_of_sym } \\
\text { metry }\end{array}$ & not covered & $\begin{array}{l}\text { AngleBetweenCharacteristicDefi } \\
\text { nitionType }\end{array}$ \\
\hline \multicolumn{4}{|l|}{ dimension tolerance principle } \\
\hline independency & $\begin{array}{l}\text { shape_dimension_representation.nam } \\
\text { e = "independency" }\end{array}$ & not covered & $\begin{array}{l}\text { <>EnvelopeRequirement(FALSE } \\
\text { ) }\end{array}$ \\
\hline envelope & $\begin{array}{l}\text { shape_dimension_representation.nam } \\
\text { e = "envelope" }\end{array}$ & not covered & <>EnvelopeRequirement(TRUE) \\
\hline \multicolumn{4}{|l|}{ dimension values } \\
\hline nominal value & $\begin{array}{l}\text { measure_representation_item.name = } \\
\text { "nominal value" }\end{array}$ & dimension value & <>TargetValue \\
\hline nominal value with qualifier & $\begin{array}{l}\text { qualified_representation_item/type_qu } \\
\text { alifier.name = } \\
\text { "maximum"/"minimum"/"average" }\end{array}$ & not covered & $<>$ TargetValue \\
\hline nominal value with plus/minus bounds & plus_minus_tolerance & not covered & <>DefinedAsLimit(FALSE) \\
\hline value range & $\begin{array}{l}\text { measure_representation_item.name = } \\
\text { "upper limit"/"lower limit"- }\end{array}$ & dimtol lower limit/dimtol upper limit & <>DefinedAsLimit(TRUE) \\
\hline tolerance class (ISO 286) & limits_and_fits & not covered & not covered \\
\hline basic/theoretical & $\begin{array}{l}\text { descriptive_representation_item.descri } \\
\text { ption = "theoretical" }\end{array}$ & dimension_type (dimtype_basic) & <>DimensionType(BASIC) \\
\hline set & & & <>DimensionType(SET) \\
\hline reference/auxiliary & $\begin{array}{l}\text { descriptive_representation_item.descri } \\
\text { ption = "auxiliary" }\end{array}$ & dimension_type (dimtype_reference) & $\begin{array}{l}\text { <>DimensionType(REFERENCE } \\
\text { ) }\end{array}$ \\
\hline key characteristic & & & <>KeyCharacteristic \\
\hline \multirow[t]{2}{*}{ non-tolerance } & & & <>NonTolerance \\
\hline & $\begin{array}{l}\text { START ITEMS FROM TABLE } 8 \text { OF } \\
\text { AP242 RECOMMENDED PRACTICES } \\
\text { (from ISO ?) }\end{array}$ & & \\
\hline
\end{tabular}




\begin{tabular}{|c|c|c|c|}
\hline PMI & STEP AP242 & $\underline{\mathrm{ACIS}}$ & $\underline{\text { QIF }}$ \\
\hline controlled radius & $\begin{array}{l}\text { descriptive_representation_item.descri } \\
\text { ption = "controlled radius" }\end{array}$ & not covered & $\begin{array}{l}\text { RadiusCharacteristicDefinitionTy } \\
\text { pe }<>\text { ControlledRadius(TRUE) }\end{array}$ \\
\hline square & $\begin{array}{l}\text { descriptive_representation_item.descri } \\
\text { ption = "square" }\end{array}$ & not covered & $\begin{array}{l}\text { SquareCharacteristicDefinitionTy } \\
\text { pe }\end{array}$ \\
\hline statistical tolerance & $\begin{array}{l}\text { descriptive_representation_item.descri } \\
\text { ption = "statistical tolerance" }\end{array}$ & dimension_type (dimtype_tolerance) & $\begin{array}{l}\text { CharacteristicDefinitionBaseType } \\
<>\text { StatisticalCharacteristic(TRUE } \\
\text { ) }\end{array}$ \\
\hline continuous feature & $\begin{array}{l}\text { descriptive_representation_item.descri } \\
\text { ption = "continuous feature" }\end{array}$ & not covered & UnitedFeature \\
\hline two point size & $\begin{array}{l}\text { descriptive_representation_item.descri } \\
\text { ption = "two point size" (not Y14.5) }\end{array}$ & not covered & $\begin{array}{l}\text { CharacteristicDirectionalZoneLim } \\
\text { itType<>FromPoint, <> ToPoint }\end{array}$ \\
\hline local size defined by a sphere & $\begin{array}{l}\text { descriptive_representation_item.descri } \\
\text { ption = "local size defined by sphere" } \\
\text { (not Y14.5) }\end{array}$ & not covered & $\begin{array}{l}\text { CharacteristicBaseType<>Descri } \\
\text { ption }\end{array}$ \\
\hline least-squares association criterion & $\begin{array}{l}\text { descriptive_representation_item.descri } \\
\text { ption = "least-squares association } \\
\text { criterion" }\end{array}$ & not covered & $\begin{array}{l}\text { XXXSubst.Feat.Algor.EnumType } \\
\text { (LEASTSQUARES), for } \\
\text { [Non]FeatureOfSize, Curve, } \\
\text { Surface }\end{array}$ \\
\hline $\begin{array}{l}\text { maximum inscribed association } \\
\text { criterion }\end{array}$ & $\begin{array}{l}\text { descriptive_representation_item.descri } \\
\text { ption = "maximum inscribed } \\
\text { association criterion" }\end{array}$ & not covered & $\begin{array}{l}\text { FeatureOfSizeSubstituteFeature } \\
\text { AlgorithmEnumType(MAXINSCR } \\
\text { IBED) }\end{array}$ \\
\hline $\begin{array}{l}\text { minimum circumscribed association } \\
\text { criterion }\end{array}$ & $\begin{array}{l}\text { descriptive_representation_item.descri } \\
\text { ption = "minimum circumscribed } \\
\text { association criterion" }\end{array}$ & not covered & $\begin{array}{l}\text { FeatureOfSizeSubstituteFeature } \\
\text { AlgorithmEnumType(MINCIRCU } \\
\text { MSCRIBED) }\end{array}$ \\
\hline circumference diameter & $\begin{array}{l}\text { descriptive_representation_item.descri } \\
\text { ption = "circumference diameter" (not } \\
\text { Y14.5) }\end{array}$ & not covered & $\begin{array}{l}\text { CharacteristicBaseType<>Descri } \\
\text { ption }\end{array}$ \\
\hline area diameter & $\begin{array}{l}\text { descriptive_representation_item.descri } \\
\text { ption = "area diameter" (not Y14.5) }\end{array}$ & not covered & $\begin{array}{l}\text { CharacteristicBaseType<>Descri } \\
\text { ption }\end{array}$ \\
\hline volume diameter & $\begin{array}{l}\text { descriptive_representation_item.descri } \\
\text { ption = "volume diameter" (not Y14.5) }\end{array}$ & not covered & $\begin{array}{l}\text { CharacteristicBaseType<>Descri } \\
\text { ption }\end{array}$ \\
\hline maximum size & $\begin{array}{l}\text { descriptive_representation_item.descri } \\
\text { ption = "maximum size" }\end{array}$ & not covered & $\begin{array}{l}\text { <>MeasurementDirectiveEnum( } \\
\text { MAXIMUM) }\end{array}$ \\
\hline minimum size & $\begin{array}{l}\text { descriptive_representation_item.descri } \\
\text { ption = "minimum size" }\end{array}$ & not covered & $\begin{array}{l}\text { <>MeasurementDirectiveEnum( } \\
\text { MINIMUM) }\end{array}$ \\
\hline average size & $\begin{array}{l}\text { descriptive_representation_item.descri } \\
\text { ption = "average size" }\end{array}$ & not covered & $\begin{array}{l}\text { <>MeasurementDirectiveEnum(A } \\
\text { VERAGE) }\end{array}$ \\
\hline median size & $\begin{array}{l}\text { descriptive_representation_item.descri } \\
\text { ption = "median size" }\end{array}$ & not covered & $\begin{array}{l}\text { CharacteristicDefinitionBaseType } \\
<>\text { MedianFeature(TRUE) }\end{array}$ \\
\hline mid-range size & $\begin{array}{l}\text { descriptive_representation_item.descri } \\
\text { ption = "mid-range size" }\end{array}$ & not covered & $\begin{array}{l}\text { CharacteristicBaseType<>Descri } \\
\text { ption }\end{array}$ \\
\hline
\end{tabular}




\begin{tabular}{|c|c|c|c|}
\hline$\underline{\text { PMI }}$ & STEP AP242 & ACIS & $\underline{\text { QIF }}$ \\
\hline range of sizes & $\begin{array}{l}\text { descriptive_representation_item.descri } \\
\text { ption = "range of sizes" }\end{array}$ & not covered & $\begin{array}{l}\text { CharacteristicBaseType }<>\text { Descri } \\
\text { ption }\end{array}$ \\
\hline any restricted portion of feature & $\begin{array}{l}\text { descriptive_representation_item.descri } \\
\text { ption = "any restricted portion of } \\
\text { feature" }\end{array}$ & not covered & $\begin{array}{l}\text { CharacteristicBaseType<>Descri } \\
\text { ption }\end{array}$ \\
\hline any cross section & $\begin{array}{l}\text { descriptive_representation_item.descri } \\
\text { ption = "any cross section" }\end{array}$ & not covered & SectionModifierEnumType(ACS) \\
\hline specific fixed cross section & $\begin{array}{l}\text { descriptive_representation_item.descri } \\
\text { ption = "specific fixed cross section" }\end{array}$ & not covered & $\begin{array}{l}\text { CharacteristicBaseType<>Descri } \\
\text { ption }\end{array}$ \\
\hline common tolerance & $\begin{array}{l}\text { descriptive_representation_item.descri } \\
\text { ption = "common tolerance" }\end{array}$ & not covered & $\begin{array}{l}\text { CharacteristicDefinitionBaseType } \\
<>\text { CommonZone(TRUE) }\end{array}$ \\
\hline \multirow[t]{2}{*}{ free-state condition } & $\begin{array}{l}\text { descriptive_representation_item.descri } \\
\text { ption = "free-state condition" }\end{array}$ & not covered & $\begin{array}{l}\text { CharacteristicDefinitionBaseType } \\
<>\text { FreeState(TRUE) }\end{array}$ \\
\hline & END TABLE 8 ITEMS & & \\
\hline united feature & $\begin{array}{l}\text { descriptive_representation_item.descri } \\
\text { ption = "continuous feature" }\end{array}$ & not covered & $\begin{array}{l}\text { CharacteristicDefinitionBaseType } \\
<>\text { UnitedFeature(TRUE) }\end{array}$ \\
\hline dimension decimal places & value_format_type_qualifier & dimtol precision & $\begin{array}{l}\text { <>SpecifiedDecimalType@decim } \\
\text { alPlaces }\end{array}$ \\
\hline datum & datum & spaxpmi_datum & DatumDefinitionType \\
\hline datum feature & datum_feature & attrib_spaxpmi_datum & FeatureNominal \\
\hline datum target & placed_datum_target_feature & spaxpmi_datumtgt & DatumTargetDefinitionBaseType \\
\hline point & $\begin{array}{l}\text { axis2_placement_3d.name = } \\
\text { "orientation" }\end{array}$ & datum_target_type (dt_point) & DatumTargetPointDefinitionType \\
\hline line & $\begin{array}{l}\text { axis2_placement_3d/length_measure_ } \\
\text { with_unit.name = "target length" }\end{array}$ & datum_target_type (dt_line) & DatumTargetLineDefinitionType \\
\hline circular line & $\begin{array}{l}\text { axis2_placement_3d/length_measure_- } \\
\text { with_unit.name = "circle diameter" }\end{array}$ & & $\begin{array}{l}\text { DatumTargetCircularLineDefinitio } \\
\text { nType }\end{array}$ \\
\hline rectangle & $\begin{array}{l}\text { axis2_placement_3d/length_measure_ } \\
\text { with_unit.name = "target width" }\end{array}$ & datum_target_type (dt_area_rect) & $\begin{array}{l}\text { DatumTargetRectangularAreaDe } \\
\text { finitionType }\end{array}$ \\
\hline circle & $\begin{array}{l}\text { axis2_placement_3d/length_measure_ } \\
\text { with_unit.name = "target diameter" }\end{array}$ & datum_target_type (dt_area_circ) & $\begin{array}{l}\text { DatumTargetCircularAreaDefiniti } \\
\text { onType }\end{array}$ \\
\hline cylindrical area & advanced_face & datum_target_type (dt_area_face) & $\begin{array}{l}\text { DatumTargetCylindricalAreaDefi } \\
\text { nitionType }\end{array}$ \\
\hline sphere & advanced_face & datum_target_type (dt_area_face) & $\begin{array}{l}\text { DatumTargetSphereDefinitionTy } \\
\text { pe }\end{array}$ \\
\hline area & advanced_face & datum_target_type (dt_area_face) & $\begin{array}{l}\text { DatumTargetlrregularAreaDefiniti } \\
\text { onType }\end{array}$ \\
\hline movable datum target & direction & not covered & $\begin{array}{l}\text { MovableDatumTargetDirectionTy } \\
\text { pe }\end{array}$ \\
\hline tolerance & geometric_tolerance & attrib_spaxpmi_geom_tol & CharacteristicDefinitionBaseType \\
\hline
\end{tabular}




\begin{tabular}{|c|c|c|c|}
\hline PMI & STEP AP242 & $\underline{\text { ACIS }}$ & $\underline{\text { QIF }}$ \\
\hline \multicolumn{4}{|l|}{ tolerance types } \\
\hline angularity & angularity_tolerance & tol_type (toltype_angularity) & $\begin{array}{l}\text { AngularityCharacteristicDefinition } \\
\text { Type }\end{array}$ \\
\hline circular runout & circular_runout_tolerance & tol_type (toltype_runout_circular) & $\begin{array}{l}\text { CircularRunoutCharacteristicDefi } \\
\text { nitionType }\end{array}$ \\
\hline circularity/roundness & roundness_tolerance & tol_type (toltype_circularity) & $\begin{array}{l}\text { CircularityCharacteristicDefinition } \\
\text { Type }\end{array}$ \\
\hline coaxiality & coaxiality_tolerance & not covered & $\begin{array}{l}{ }^{*} \text { ConcentricityCharacteristicDefin } \\
\text { itionType }\end{array}$ \\
\hline concentricity & concentricity_tolerance & tol_type (toltype_concentricity) & $\begin{array}{l}\text { ConcentricityCharacteristicDefinit } \\
\text { ionType }\end{array}$ \\
\hline cylindricity & cylindricity_tolerance & tol_type (toltype_cylindricity) & $\begin{array}{l}\text { CylindricityCharacteristicDefinitio } \\
\text { nType }\end{array}$ \\
\hline flatness & flatness_tolerance & tol_type (toltype_flatness) & $\begin{array}{l}\text { FlatnessCharacteristicDefinitionT } \\
\text { ype }\end{array}$ \\
\hline parallelism & parallelism_tolerance & tol_type (toltype_parallelism) & $\begin{array}{l}\text { ParallelismCharacteristicDefinitio } \\
\text { nType }\end{array}$ \\
\hline perpendicularity & perpendicularity_tolerance & tol_type (toltype_perpendicularity) & $\begin{array}{l}\text { PerpendicularityCharacteristicDe } \\
\text { finitionType }\end{array}$ \\
\hline position & position_tolerance & tol_type (toltype_position) & $\begin{array}{l}\text { PositionCharacteristicDefinitionT } \\
\text { ype }\end{array}$ \\
\hline profile of a line & line_profile_tolerance & tol_type (toltype_profile_line) & $\begin{array}{l}\text { LineProfileCharacteristicDefinitio } \\
\text { nType }\end{array}$ \\
\hline profile of a point & & & $\begin{array}{l}\text { PointProfileCharacteristicDefiniti } \\
\text { onType }\end{array}$ \\
\hline profile of a surface & surface_profile_tolerance & tol_type (toltype_profile_surf) & $\begin{array}{l}\text { SurfaceProfileCharacteristicDefin } \\
\text { itionType }\end{array}$ \\
\hline straightness & straightness_tolerance & tol_type (toltype_straightness) & $\begin{array}{l}\text { StraightnessCharacteristicDefiniti } \\
\text { onType }\end{array}$ \\
\hline symmetry & symmetry_tolerance & tol_type (toltype_symmetry) & $\begin{array}{l}\text { SymmetryCharacteristicDefinition } \\
\text { Type }\end{array}$ \\
\hline texture & & & $\begin{array}{l}\text { SurfaceTextureCharacteristicDefi } \\
\text { nitionType }\end{array}$ \\
\hline thread & & & $\begin{array}{l}\text { ThreadCharacteristicDefinitionTy } \\
\text { pe }\end{array}$ \\
\hline total runout & total_runout_tolerance & tol_type (toltype_runout_total) & $\begin{array}{l}\text { TotalRunoutCharacteristicDefiniti } \\
\text { onType }\end{array}$ \\
\hline \multicolumn{4}{|l|}{ tolerance zone } \\
\hline diameter & $\begin{array}{l}\text { tolerance_zone_form.name = } \\
\text { "cylindrical or circular" }\end{array}$ & mod_dia_type (dm_dia) & $<>$ DiametricalZone \\
\hline
\end{tabular}




\begin{tabular}{|c|c|c|c|}
\hline PMI & STEP AP242 & $\underline{\text { ACIS }}$ & $\underline{\text { QIF }}$ \\
\hline spherical diameter & $\begin{array}{l}\text { tolerance_zone_form.name = } \\
\text { "spherical" }\end{array}$ & mod_dia_type (dm_spherical_dia) & <>SphericalZone \\
\hline within a circle & $\begin{array}{l}\text { tolerance_zone_form.name = "within a } \\
\text { circle" }\end{array}$ & not covered & $\begin{array}{l}\text { CharacteristicCircularZoneLimitT } \\
\text { ype }\end{array}$ \\
\hline rectangular tolerance zone & $\begin{array}{l}\text { tolerance_zone_form.name = } \\
\text { "rectangular" }\end{array}$ & not covered & $\begin{array}{l}\text { CharacteristicRectangularZoneLi } \\
\text { mitType }\end{array}$ \\
\hline directional tolerance zone & $\begin{array}{l}\text { tolerance_zone_form.name = } \\
\text { "directional" }\end{array}$ & not covered & $\begin{array}{l}\text { CharacteristicDirectionalZoneLim } \\
\text { itType }\end{array}$ \\
\hline between two concentric circles & $\begin{array}{l}\text { tolerance_zone_form.name }= \\
\text { "between two concentric circles" }\end{array}$ & not covered & ZoneRadiiType \\
\hline between two equidistant curves & $\begin{array}{l}\text { tolerance_zone_form.name = } \\
\text { "between two equidistant curves" }\end{array}$ & not covered & $\begin{array}{l}\text { <>UnequallyDisposedZone [also } \\
\text { explicit in Straightness, } \\
\text { Circularity] }\end{array}$ \\
\hline within a cylinder & $\begin{array}{l}\text { tolerance_zone_form.name = "within a } \\
\text { cylinder" }\end{array}$ & not covered & $\begin{array}{l}\text { CylindricityCharacteristicNominal } \\
\text { Type }<>\text { CharacteristicDirectional } \\
\text { ZoneLimitType }\end{array}$ \\
\hline between two coaxial cylinders & $\begin{array}{l}\text { tolerance_zone_form.name = } \\
\text { "between two coaxial cylinders" }\end{array}$ & not covered & $\begin{array}{l}\text { ConcentricityCharacteristicNomin } \\
\text { alType<> ZoneLimit }\end{array}$ \\
\hline between two equidistant surfaces & $\begin{array}{l}\text { tolerance_zone_form.name = } \\
\text { "between two equidistant surfaces" }\end{array}$ & not covered & $\begin{array}{l}\text { ConcentricityNonDiametricalZon } \\
\text { eType [implicit in SurfaceProfile } \\
\text { \& Flatness, explicit in Cylindricity] }\end{array}$ \\
\hline runout & runout_zone_definition & not covered & $\begin{array}{l}\text { RunoutCharacteristicNominalBas } \\
\text { eType }<>\text { ZoneDirection }\end{array}$ \\
\hline projected & projected_zone_definition & p_mag & <>ProjectedToleranceZoneValue \\
\hline non-uniform & non_uniform_zone_definition & not covered & $\begin{array}{l}\text { SurfaceProfileNonUniformChara } \\
\text { cteristicDefinitionType }\end{array}$ \\
\hline non-diametrical & & & $\begin{array}{l}\text { <>NonDiametricalZone [position, } \\
\text { straightness, concentricity] }\end{array}$ \\
\hline elongated & & & $\begin{array}{l}\text { <>ElongatedZone [in } \\
\text { PositionDiametricalZoneType] }\end{array}$ \\
\hline outer disposition & & & <>OuterDisposition \\
\hline offest & & & <>OffsetZone \\
\hline planar & & & <>PlanarZone [orientation] \\
\hline \multicolumn{4}{|l|}{$\begin{array}{l}\text { tolerance modifiers (from RecPrac } \\
6.9 .3 \text { ) }\end{array}$} \\
\hline any cross section & $\begin{array}{l}\text { geometric_tolerance_with_modifiers.m } \\
\text { odifiers =.ANY_CROSS_SECTION. }\end{array}$ & not covered & $<>$ IntersectionPlane \\
\hline common zone & $\begin{array}{l}\text { geometric_tolerance_with_modifiers.m } \\
\text { odifiers = .COMMON_ZONE. }\end{array}$ & not covered & <>CommonZone(TRUE) \\
\hline
\end{tabular}




\begin{tabular}{|c|c|c|c|}
\hline PMI & STEP AP242 & ACIS & $\underline{\text { QIF }}$ \\
\hline each radial element & $\begin{array}{l}\text { geometric_tolerance_with_modifiers.m } \\
\text { odifiers = .EACH_RADIAL_ELEMENT. }\end{array}$ & not covered & $\begin{array}{l}\text { OrientationCharacteristicDefinitio } \\
\text { nBaseType<> EachRadialElemen } \\
\mathrm{t}(\text { TRUE) }\end{array}$ \\
\hline free state & $\begin{array}{l}\text { geometric_tolerance_with_modifiers.m } \\
\text { odifiers = .FREE_STATE. }\end{array}$ & zone_modifier_type (zm_fs) & $<>$ FreeState(TRUE) \\
\hline least material requirement & $\begin{array}{l}\text { geometric_tolerance_with_modifiers.m } \\
\text { odifiers = } \\
\text {.LEAST_MATERIAL_REQUIREMENT. }\end{array}$ & zone_modifier_type (zm_Imc) & <>MaterialCondition(LEAST) \\
\hline line element & $\begin{array}{l}\text { geometric_tolerance_with_modifiers.m } \\
\text { odifiers }=\text {.LINE_ELEMENT. }\end{array}$ & not covered & <>EachElement(TRUE) \\
\hline major diameter (of thread) & $\begin{array}{l}\text { geometric_tolerance_with_modifiers.m } \\
\text { odifiers = .MAJOR_DIAMETER. }\end{array}$ & not covered & not covered \\
\hline maximum material requirement & $\begin{array}{l}\text { geometric_tolerance_with_modifiers.m } \\
\text { odifiers = } \\
\text {.MAXIMUM_MATERIAL_REQUIREME } \\
\text { NT. }\end{array}$ & zone_modifier_type (zm_mmc) & <>MaterialCondition(MAXIMUM) \\
\hline minor diameter (of thread) & $\begin{array}{l}\text { geometric_tolerance_with_modifiers.m } \\
\text { odifiers }=\text {.MINOR_DIAMETER. }\end{array}$ & not covered & not covered \\
\hline not convex & $\begin{array}{l}\text { geometric_tolerance_with_modifiers.m } \\
\text { odifiers }=. \text { NOT_CONVEX. }\end{array}$ & not covered & $\begin{array}{l}\text { FlatnessCharacteristicDefinitionT } \\
\text { ype<>NotConvex(TRUE) }\end{array}$ \\
\hline pitch diameter & $\begin{array}{l}\text { geometric_tolerance_with_modifiers.m } \\
\text { odifiers = .PITCH_DIAMETER. }\end{array}$ & not covered & $\begin{array}{l}\text { ThreadCharacteristicActualType } \\
\text { <>PitchDiameter }\end{array}$ \\
\hline reciprocity requirement & $\begin{array}{l}\text { geometric_tolerance_with_modifiers.m } \\
\text { odifiers = } \\
\text {.RECIPROCITY_REQUIREMENT. }\end{array}$ & zone_modifier_type (zm_rfs) & not covered \\
\hline separate requirement & $\begin{array}{l}\text { geometric_tolerance_with_modifiers.m } \\
\text { odifiers = } \\
\text {.SEPARATE_REQUIREMENT. }\end{array}$ & not covered & <>SeparateZone(TRUE) \\
\hline statistical tolerance & $\begin{array}{l}\text { geometric_tolerance_with_modifiers.m } \\
\text { odifiers = } \\
\text {.STATISTICAL_TOLERANCE. }\end{array}$ & zone_modifier_type (zm_st) & $\begin{array}{l}\text { <>StatisticalCharacteristic(TRUE } \\
\end{array}$ \\
\hline tangent plane & $\begin{array}{l}\text { geometric_tolerance_with_modifiers.m } \\
\text { odifiers = .TANGENT_PLANE. }\end{array}$ & zone_modifier_type (zm_tp) & <>TangentPlane(TRUE) \\
\hline collection plane & & & $<>$ CollectionPlane \\
\hline direction feature & & & <>DirectionFeature \\
\hline orientation plane & & & <>OrientationPlane \\
\hline orientation only & & & <>OrientationOnly(TRUE) \\
\hline unequally-disposed tolerance & $\begin{array}{l}\text { unequally_disposed_geometric_tolera } \\
\text { nce }\end{array}$ & p_shift & <>UnequallyDisposedZone \\
\hline tolerance with maximum value & $\begin{array}{l}\text { geometric_tolerance_with_maximum_t } \\
\text { olerance }\end{array}$ & not covered & <>MaximumToleranceValue \\
\hline
\end{tabular}




\begin{tabular}{|c|c|c|c|}
\hline PMI & STEP AP242 & $\underline{\mathrm{ACIS}}$ & $\underline{\text { QIF }}$ \\
\hline \multicolumn{4}{|l|}{ unit-basis tolerance } \\
\hline length & $\begin{array}{l}\text { geometric_tolerance_with_defined_uni } \\
\mathrm{t}\end{array}$ & runit1 & $\begin{array}{l}\text { ToleranceZonePerUnitLengthTyp } \\
\mathrm{e}\end{array}$ \\
\hline circular & $\begin{array}{l}\text { geometric_tolerance_with_defined_are } \\
\text { a_unit.area_type }=. \bar{C} \text { IRCULAR. }\end{array}$ & runit1 & ToleranceZonePerUnitArcLength \\
\hline rectangular & $\begin{array}{l}\text { geometric_tolerance_with_defined_are } \\
\text { a_unit.area_type }=. \text { RECTANGULAR. }\end{array}$ & runit1,runit2 & ToleranceZonePerUnitAreaType \\
\hline square & $\begin{array}{l}\text { geometric_tolerance_with_defined_are } \\
\text { a_unit.area_type }=. \text { SQUARE. }\end{array}$ & runit1 & ToleranceZonePerUnitAreaType \\
\hline per-unit-polar-area & not covered & not covered & $\begin{array}{l}\text { ToleranceZonePerUnitPolarArea } \\
\text { Type }\end{array}$ \\
\hline per-unit-angle & not covered & not covered & $\begin{array}{l}\text { ToleranceZonePerUnitAngleTyp } \\
\text { e }\end{array}$ \\
\hline composite tolerance & geometric_tolerance_relationship & attrib_spaxpmi_geom_tol & $\begin{array}{l}\text { CompositeSegmentDefinitionTyp } \\
\text { eBase }\end{array}$ \\
\hline tolerance with datum references & $\begin{array}{l}\text { geometric_tolerance_with_datum_refer } \\
\text { ence }\end{array}$ & spaxpmi_drf & $X X X<>$ DatumReferenceFrameld \\
\hline datum reference & datum_reference_compartment & spaxpmi_dref & $\begin{array}{l}\text { <>DatumReferenceFrame } \\
\text { <>Datum }\end{array}$ \\
\hline \multicolumn{4}{|l|}{ datum reference modifiers } \\
\hline free state & $\begin{array}{l}\text { simple_datum_reference_modifier.mod } \\
\text { ifiers = .FREE_STATE. }\end{array}$ & not covered & $<>$ FreeState \\
\hline basic & $\begin{array}{l}\text { simple_datum_reference_modifier.mod } \\
\text { ifiers = .BASIC. }\end{array}$ & not covered & $\begin{array}{l}\text { DatumFeatureSimulatorModifierT } \\
\text { ype<>BasicSize("BASIC") }\end{array}$ \\
\hline diametrical & & & $\begin{array}{l}\text { DatumFeatureSimulatorModifierT } \\
\text { ype<>DiametricalSize }\end{array}$ \\
\hline linear & & & $\begin{array}{l}\text { DatumFeatureSimulatorModifierT } \\
\text { ype }<>\text { LinearSize }\end{array}$ \\
\hline translation & $\begin{array}{l}\text { simple_datum_reference_modifier.mod } \\
\text { ifiers = .TRANSLATION. }\end{array}$ & not covered & $\begin{array}{l}\text { DatumTranslationType<>Datum } \\
\text { Translation }\end{array}$ \\
\hline least material requirement & $\begin{array}{l}\text { simple_datum_reference_modifier.mod } \\
\text { ifiers = } \\
\text {.LEAST_MATERIAL_REQUIREMENT. }\end{array}$ & $\begin{array}{l}\text { datum_modifier_type } \\
\text { (datum_modifier_Imc) }\end{array}$ & <>MaterialModifier(LEAST) \\
\hline maximum material requirement & $\begin{array}{l}\text { simple_datum_reference_modifier.mod } \\
\text { ifiers = } \\
\text {.MAXIMUM_MATERIAL_REQUIREME } \\
\text { NT. }\end{array}$ & $\begin{array}{l}\text { datum_modifier_type } \\
\text { (datum_modifier_mmc) }\end{array}$ & <>MaterialModifier(MAXIMUM) \\
\hline regardless & & & $\begin{array}{l}<>\text { MaterialModifier(REGARDLES } \\
\text { S) }\end{array}$ \\
\hline point & $\begin{array}{l}\text { simple_datum_reference_modifier.mod } \\
\text { ifiers }=\text {.POINT. }\end{array}$ & not covered & <>ReducedDatum(PT) \\
\hline
\end{tabular}




\begin{tabular}{|c|c|c|c|}
\hline$\underline{\text { PMI }}$ & STEP AP242 & $\underline{\text { ACIS }}$ & $\underline{\text { QIF }}$ \\
\hline line & $\begin{array}{l}\text { simple_datum_reference_modifier.mod } \\
\text { ifiers = .LINE. }\end{array}$ & not covered & $<>$ ReducedDatum(SL) \\
\hline plane & $\begin{array}{l}\text { simple_datum_reference_modifier.mod } \\
\text { ifiers }=\text {.PLANE. }\end{array}$ & not covered & $<>$ ReducedDatum(PL) \\
\hline orientation & $\begin{array}{l}\text { simple_datum_reference_modifier.mod } \\
\text { ifiers =-ORIENTATION. }\end{array}$ & not covered & <>ConstrainOrientation(TRUE) \\
\hline any cross section & $\begin{array}{l}\text { simple_datum_reference_modifier.mod } \\
\text { ifiers }=\text {.ANY_CROSS_SECTION. }\end{array}$ & not covered & <>SectionModifier(ACS) \\
\hline any longitudinal section & $\begin{array}{l}\text { simple_datum_reference_modifier.mod } \\
\text { ifiers = } \\
\text {.ANY_LONGITUDINAL_SECTION. }\end{array}$ & not covered & <>SectionModifier(ALS) \\
\hline contacting feature & $\begin{array}{l}\text { simple_datum_reference_modifier.mod } \\
\text { ifiers =.CONTACTING_FEATURE. }\end{array}$ & not covered & <>ContactingFeature(TRUE) \\
\hline distance variable & $\begin{array}{l}\text { simple_datum_reference_modifier.mod } \\
\text { ifiers = .DISTANCE_VARIABLE. }\end{array}$ & not covered & $<>$ DistanceVariable(TRUE) \\
\hline constrain orientation & & & <>ConstrainOrientation(TRUE) \\
\hline constrain subsequent & & & <>ConstrainSubsequent(TRUE) \\
\hline fixed & & & <>DatumFixed(TRUE) \\
\hline degree of freedom constraint $x$ & $\begin{array}{l}\text { simple_datum_reference_modifier.mod } \\
\text { ifiers = } \\
\text {.DEGREE_OF_FREEDOM_CONSTR } \\
\text { AINT_X. }\end{array}$ & not covered & DegreeOfFreedomEnumType(X) \\
\hline degree of freedom constraint $y$ & $\begin{array}{l}\text { simple_datum_reference_modifier.mod } \\
\text { ifiers = } \\
\text {.DEGREE_OF_FREEDOM_CONSTR } \\
\text { AINT_Y. }\end{array}$ & not covered & DegreeOfFreedomEnumType(Y) \\
\hline degree of freedom constraint $z$ & $\begin{array}{l}\text { simple_datum_reference_modifier.mod } \\
\text { ifiers = } \\
\text {.DEGREE_OF_FREEDOM_CONSTR } \\
\text { AINT_Z. }\end{array}$ & not covered & DegreeOfFreedomEnumType(Z) \\
\hline degree of freedom constraint $u$ & $\begin{array}{l}\text { simple_datum_reference_modifier.mod } \\
\text { ifiers = } \\
\text {.DEGREE_OF_FREEDOM_CONSTR } \\
\text { AINT_U. }\end{array}$ & not covered & DegreeOfFreedomEnumType(U) \\
\hline degree of freedom constraint $v$ & $\begin{array}{l}\text { simple_datum_reference_modifier.mod } \\
\text { ifiers = } \\
\text {.DEGREE_OF_FREEDOM_CONSTR } \\
\text { AINT_V. }\end{array}$ & not covered & DegreeOfFreedomEnumType(V) \\
\hline
\end{tabular}




\begin{tabular}{|c|c|c|c|}
\hline$\underline{\text { PMI }}$ & STEP AP242 & ACIS & $\underline{\text { QIF }}$ \\
\hline degree of freedom constraint $w$ & $\begin{array}{l}\text { simple_datum_reference_modifier.mod } \\
\text { ifiers = } \\
\text {.DEGREE_OF_FREEDOM_CONSTR } \\
\text { AINT_W. }\end{array}$ & not covered & DegreeOfFreedomEnumType(W) \\
\hline minor diameter & $\begin{array}{l}\text { simple_datum_reference_modifier.mod } \\
\text { ifiers }=\text {.MINOR_DIAMETER. }\end{array}$ & not covered & DiameterModifierEnumType(LD) \\
\hline major diameter & $\begin{array}{l}\text { simple_datum_reference_modifier.mod } \\
\text { ifiers =.MAJOR_DIAMETER. }\end{array}$ & not covered & DiameterModifierEnumType(MD) \\
\hline pitch diameter & $\begin{array}{l}\text { simple_datum_reference_modifier.mod } \\
\text { ifiers =.PITCH_DIAMETER. }\end{array}$ & not covered & DiameterModifierEnumType(PD) \\
\hline with value & datum_reference_modifier_with_value & not covered & $\begin{array}{l}\text { DatumFeatureSimulatorModifierT } \\
\text { ype }<>\text { LinearSize/DiametricalSize }\end{array}$ \\
\hline projected & & & <>ProjectedDatum \\
\hline $\begin{array}{l}\text { common datum/multiple datum } \\
\text { features }\end{array}$ & datum_reference_element & spaxpmi_dref & <>CompoundDatum \\
\hline polyline presentation & $\begin{array}{l}\text { annotation_curve_occurrence/annotati } \\
\text { on_fill_area_occurrence/annotation_sy } \\
\text { mbol_occurrence/annotation_text_occ } \\
\text { urrence/tessellated_annotation_occurr } \\
\text { ence }\end{array}$ & body/wire & UserDefinedExtension \\
\hline
\end{tabular}

\section{Appendix A.2: Mapping Linkages into STEP and QIF )}

\begin{tabular}{|l|l|l|l|} 
linkages & STEP AP242 & $\underline{\text { ACIS }}$ & QIF \\
\hline PMI<->BREP & $\begin{array}{l}\text { BREP<- } \\
\text { geometric_item_specific_usage- } \\
\text { >shape_aspect<-PMI }\end{array}$ & spacollection/entity \\
\hline PMI<->polyline presentation & $\begin{array}{l}\text { PMI<- } \\
\text { draughting_model_item_association- } \\
\text { >annotation_occurrence/draughting_c } \\
\text { allout }\end{array}$ & not covered & PMI->Feature->Brep \\
\hline & & PMIDisplayType->PMI & \\
\hline
\end{tabular}


Appendix A.3: Mapping PMI Features and User Defined Tolerances into STEP and QIF )

\begin{tabular}{|c|c|c|c|}
\hline PMI features & STEP AP242 & ACIS & $\underline{\text { QIF }}$ \\
\hline arc feature & not covered & & ArcFeatureXXXType \\
\hline circle feature & not covered & & CircleFeatureXXXType \\
\hline compound feature & not covered & & CompoundFeatureXXXType \\
\hline pattern feature & not covered & & PatternFeatureXXXType \\
\hline profile group feature & not covered & & ProfileGroupFeatureXXXType \\
\hline runout group feature & not covered & & RunoutGroupFeatureXXXType \\
\hline cone feature & not covered & & ConeFeatureXXXType \\
\hline conical segment feature & not covered & & $\begin{array}{l}\text { ConicalSegmentFeatureXXXTyp } \\
\text { e }\end{array}$ \\
\hline cuboid (box) feature & not covered & & CuboidFeatureXXXType \\
\hline cylinder feature & not covered & & CylinderFeatureXXXType \\
\hline cylindrical segment feature & not covered & & $\begin{array}{l}\text { CylindricalSegmentFeatureXXXT } \\
\text { ype }\end{array}$ \\
\hline edge point feature & not covered & & EdgePointFeatureXXXType \\
\hline ellipse feature & not covered & & EllipseFeatureXXXType \\
\hline elongated cylinder feature & not covered & & $\begin{array}{l}\text { ElongatedCylinderFeatureXXXTy } \\
\text { pe }\end{array}$ \\
\hline extruded cross section feature & not covered & & $\begin{array}{l}\text { ExtrudedCrossSectionFeatureXX } \\
\text { XType }\end{array}$ \\
\hline line feature & not covered & & LineFeatureXXXType \\
\hline opposite (parallel) lines feature & not covered & & OppositeLinesFeatureXXXType \\
\hline opposite (parallel) planes feature & not covered & & OppositePlanesFeatureXXXType \\
\hline plane feature & not covered & & PlaneFeatureXXXType \\
\hline point feature & not covered & & PointFeatureXXXType \\
\hline sphere feature & not covered & & SphereFeatureXXXType \\
\hline spherical segment feature & not covered & & $\begin{array}{l}\text { SphericalSegmentFeatureXXXTy } \\
\text { pe }\end{array}$ \\
\hline
\end{tabular}




\begin{tabular}{|c|c|c|c|}
\hline PMI features & STEP AP242 & $\underline{\mathrm{ACIS}}$ & $\underline{\text { QIF }}$ \\
\hline surface of revolution feature & not covered & & $\begin{array}{l}\text { SurfaceOfRevolutionFeatureXXX } \\
\text { Type }\end{array}$ \\
\hline threaded feature & not covered & & ThreadedFeatureXXXType \\
\hline toroidal segment feature & not covered & & $\begin{array}{l}\text { ToroidalSegmentFeatureXXXTyp } \\
\text { e }\end{array}$ \\
\hline torus feature & not covered & & TorusFeatureXXXType \\
\hline user defined tolerance & STEP AP242 & $\underline{\mathrm{ACIS}}$ & $\underline{\text { QIF }}$ \\
\hline angular & & & $\begin{array}{l}\text { UserDefinedAngularCharacteristi } \\
\text { cDefinitionType }\end{array}$ \\
\hline area & & & $\begin{array}{l}\text { UserDefinedAreaCharacteristicD } \\
\text { efinitionType }\end{array}$ \\
\hline attribute & & & $\begin{array}{l}\text { UserDefinedAttributeCharacterist } \\
\text { icDefinitionType }\end{array}$ \\
\hline force & & & $\begin{array}{l}\text { UserDefinedForceCharacteristic } \\
\text { DefinitionType }\end{array}$ \\
\hline linear & & & $\begin{array}{l}\text { UserDefinedLinearCharacteristic } \\
\text { DefinitionType }\end{array}$ \\
\hline mass & & & $\begin{array}{l}\text { UserDefinedMassCharacteristicD } \\
\text { efinitionType }\end{array}$ \\
\hline pressure & & & $\begin{array}{l}\text { UserDefinedPressureCharacteris } \\
\text { ticDefinitionType }\end{array}$ \\
\hline speed & & & $\begin{array}{l}\text { UserDefinedSpeedCharacteristic } \\
\text { DefinitionType }\end{array}$ \\
\hline temperature & & & $\begin{array}{l}\text { UserDefinedTemperatureCharact } \\
\text { eristicDefinitionType }\end{array}$ \\
\hline time & & & $\begin{array}{l}\text { UserDefinedTimeCharacteristicD } \\
\text { efinitionType }\end{array}$ \\
\hline unit & & & $\begin{array}{l}\text { UserDefinedUnitCharacteristicDe } \\
\text { finitionType }\end{array}$ \\
\hline
\end{tabular}


Appendix A.4: Mapping of Boundary Representation (BREP) Geometry into STEP and QIF )

\begin{tabular}{|c|c|c|c|}
\hline BREP & STEP AP242 & $\underline{\mathrm{ACIS}}$ & $\underline{\text { QIF }}$ \\
\hline \multicolumn{4}{|l|}{ topology } \\
\hline solid & manifold_solid_brep & body/lump & BodyType \\
\hline shell & closed_shell/open_shell & shell & ShellType \\
\hline face & advanced_face & face & FaceType \\
\hline mesh face & tessellated_face & not covered & FaceMeshType \\
\hline loop & $\begin{array}{l}\text { face_bound/face_outer_bound/edge_I } \\
\text { oop/vertex_loop }\end{array}$ & loop & LoopType \\
\hline mesh loop & tessellated_wire & not covered & LoopMeshType \\
\hline edge & oriented_edge/edge_curve & edge/coedge & EdgeType/CoEdgeType \\
\hline vertex & vertex_point/cartesian_point & vertex & VertexType \\
\hline \multicolumn{4}{|l|}{ surface geometry } \\
\hline cone & conical_surface & cone & Cone23Type \\
\hline cylinder & cylindrical_surface & cone & Cylinder23Type \\
\hline extruded surface & surface_of_linear_extrusion & spline & Extrude23Type \\
\hline spline surface & $\begin{array}{l}\text { b_spline_surface/b_spline_surface_wit } \\
\text { h_knots/rational_b_spline_surface/unif } \\
\text { orm_surface/quasi_uniform_surface/be } \\
\text { zier_surface }\end{array}$ & spline & Spline23Type \\
\hline nurbs & $\begin{array}{l}\text { b_spline_surface/b_spline_surface_wit } \\
\text { h_knots/rational_b_spline_surface/unif } \\
\text { orm_surface/quasi_uniform_surface/be } \\
\text { zier_surface }\end{array}$ & spline & Nurbs23Type \\
\hline offset surface & offset_surface & off_spl_sur & Offset23Type \\
\hline plane & planar_surface & plane & Plane23Type \\
\hline revolved surface & surface_of_revolution & rot_spl_sur & Revolution23Type \\
\hline sphere & spherical_surface & sphere & Sphere23Type \\
\hline torus & toroidal_surface & torus & Torus23Type \\
\hline ruled surface & $\begin{array}{l}\text { b_spline_surface/b_spline_surface_wit } \\
\text { h_knots/rational_b_spline_surface/unif } \\
\text { orm_surface/quasi_uniform_surface/be } \\
\text { zier_surface }\end{array}$ & spline & Ruled23Type \\
\hline \multicolumn{4}{|l|}{ curve geometry } \\
\hline circle & circle & ellipse & $\begin{array}{l}\text { ArcCircular13Type/ArcCircular12 } \\
\text { Type }\end{array}$ \\
\hline
\end{tabular}




\begin{tabular}{|c|c|c|c|}
\hline BREP & STEP AP242 & $\underline{\mathrm{ACIS}}$ & QIF \\
\hline ellipse & ellipse & ellipse & $\begin{array}{l}\text { ArcConic13Type(ELLIPSE)/ArcC } \\
\text { onic12Type(ELLIPSE) }\end{array}$ \\
\hline parabola & parabola & bs3_curve & $\begin{array}{l}\text { ArcConic13Type(PARABOLA)/Ar } \\
\text { cConic12Type(PARABOLA) }\end{array}$ \\
\hline hyperbola & hyperbola & bs3_curve & $\begin{array}{l}\text { ArcConic13Type(HYPERBOLA)/ } \\
\text { ArcConic12Type(HYPERBOLA) }\end{array}$ \\
\hline spline curve & $\begin{array}{l}\text { b_spline_curve/b_spline_curve_with_k } \\
\text { nots/rational_b_spline_curve/uniform_- } \\
\text { curve/quasi_uniform_curve/bezier_cur } \\
\text { ve }\end{array}$ & bs3_curve & Spline13Type/Spline12Type \\
\hline nurbs & $\begin{array}{l}\text { b_spline_curve/b_spline_curve_with_k } \\
\text { nots/rational_b_spline_curve/uniform_- } \\
\text { curve/quasi_uniform_curve/bezier_cur } \\
\text { ve }\end{array}$ & bs3_curve & Nurbs13Type/Nurbs12Type \\
\hline offset curve & offset_curve_3d & bs3_curve & Nurbs13Type/Nurbs12Type \\
\hline line & line & straight & $\begin{array}{l}\text { Segment13Type/Segment12Typ } \\
\text { e }\end{array}$ \\
\hline poly-line & polyline & body/wire & Polyline13Type/Polyline12Type \\
\hline aggregate curve & composite_curve & body/wire & $\begin{array}{l}\text { Aggregate13Type/Aggregate12T } \\
\text { ype }\end{array}$ \\
\hline
\end{tabular}

\section{Appendix A.5: Mapping Other MBD-related Items into STEP and QIF}

\section{PMI \\ STEP AP242}

Notes

Flag Notes

Surface Finish (roughness)

Tables

Global or General Tolerances

Views text_literal

not supported

not supported

not supported

not supported

draughting_model/camera_model

\section{ACIS}

not supported

attrib_spaxpmi_flagnote

attrib_spaxpmi_roughness

not supported

not supported

spaxpmi_capture

\section{QIF}

NoteType

NoteFlagType

SurfaceTextureCharacteristicDefinitionType not supported

not supported

SavedViewType 


\section{Appendix B: Validation Results for Test Models}

\section{Appendix B.1: Validation Differences by PMI Category for Test Models}

\begin{tabular}{|c|c|c|c|c|c|c|c|c|c|c|c|c|c|c|c|}
\hline STEP Model File & $\begin{array}{l}\text { Native } \\
\text { CAD }\end{array}$ & $\begin{array}{l}\text { STEP } \\
\text { Vendor }\end{array}$ & Date & $\begin{array}{c}\text { DFS } \\
\text { Count }\end{array}$ & $\begin{array}{c}\text { DTS } \\
\text { Count }\end{array}$ & $\begin{array}{c}\text { DIM } \\
\text { Count }\end{array}$ & $\begin{array}{c}\text { FCF } \\
\text { Count }\end{array}$ & $\begin{array}{l}\text { NOTE } \\
\text { Count }\end{array}$ & $\begin{array}{l}\text { Total } \\
\text { Count }\end{array}$ & $\begin{array}{l}\text { DFS } \\
\text { Diffs }\end{array}$ & $\begin{array}{l}\text { DTS } \\
\text { Diffs }\end{array}$ & $\begin{array}{c}\text { DIM } \\
\text { Diffs }\end{array}$ & $\begin{array}{l}\text { FCF } \\
\text { Diffs } \\
\end{array}$ & $\begin{array}{c}\text { NOTE } \\
\text { Diffs }\end{array}$ & $\begin{array}{l}\text { Total } \\
\text { Diffs }\end{array}$ \\
\hline 827-9999-903.stp & $\mathrm{NX}$ & CoreTech & $04 / 28 / 15$ & 2 & & 8 & 6 & 7 & 23 & & & 5 & & 7 & 12 \\
\hline 827-9999-904.stp & $\mathrm{NX}$ & CoreTech & $07 / 10 / 15$ & 3 & & 54 & 13 & 8 & 78 & & & 1 & & 8 & 9 \\
\hline nist_ctc_01_asme1_ct5210_rd_ct242repr.stp & CATIA & CoreTech & 12/19/14 & 3 & & 10 & 6 & 3 & 22 & 1 & & 3 & & 3 & 7 \\
\hline nist_ctc_01_asme1_ct5210_rd_dk242repr.stp & CATIA & Datakit & $02 / 16 / 15$ & 3 & & 10 & 6 & 3 & 22 & & & 4 & & 3 & 7 \\
\hline nist_ctc_01_asme1_nx800_rd_ct242repr.stp & $\mathrm{NX}$ & CoreTech & $02 / 18 / 15$ & 3 & & 10 & 6 & 2 & 21 & & & 3 & & 2 & 5 \\
\hline nist_ctc_01_asme1_nx800_rd_nx.stp & $\mathrm{NX}$ & SPLM & $11 / 04 / 15$ & 3 & & 10 & 6 & 2 & 21 & & & 9 & & 2 & 11 \\
\hline nist_ctc_01_asme1_nx800_rd_th.stp & $\mathrm{NX}$ & Theorem & 11/10/15 & 3 & & 10 & 6 & 2 & 21 & & & 1 & & 2 & 3 \\
\hline nist_ctc_02_asme1_ct5210_rc_ct242repr.stp & CATIA & CoreTech & 12/19/14 & 10 & 9 & 8 & 22 & 1 & 50 & 10 & 9 & 8 & 22 & 1 & 50 \\
\hline nist_ctc_02_asme1_ct5210_rc_dk242repr.stp & CATIA & Datakit & $02 / 16 / 15$ & 10 & 9 & 8 & 22 & 1 & 50 & 10 & 9 & 8 & 22 & 1 & 50 \\
\hline nist_ctc_02_asme1_nx800_rc_ct242repr.stp & $\mathrm{NX}$ & CoreTech & $02 / 18 / 15$ & 7 & 9 & 7 & 22 & 1 & 46 & 7 & 9 & 7 & 22 & 1 & 46 \\
\hline nist_ctc_02_asme1_nx800_rc_nx.stp & $\mathrm{NX}$ & SPLM & $11 / 04 / 15$ & 6 & 9 & 7 & 22 & 2 & 46 & & 9 & & & 2 & 11 \\
\hline nist_ctc_02_asme1_nx800_rc_th.stp & $\mathrm{NX}$ & Theorem & 11/10/15 & 10 & 9 & 7 & 22 & 1 & 49 & 10 & 9 & 7 & 22 & 1 & 49 \\
\hline nist_ctc_03_asme1_ct5210_rc_ct242repr.stp & CATIA & CoreTech & $12 / 19 / 14$ & 6 & & 10 & 13 & 1 & 30 & & & 2 & & 1 & 3 \\
\hline nist_ctc_03_asme1_ct5210_rc_dk242repr.stp & CATIA & Datakit & $02 / 16 / 15$ & 6 & & 10 & 13 & 1 & 30 & & & 2 & & 1 & 3 \\
\hline nist_ctc_03_asme1_nx800_rc_ct242repr.stp & $\mathrm{NX}$ & CoreTech & $02 / 18 / 15$ & 6 & & 10 & 13 & & 29 & & & 2 & & & 2 \\
\hline nist_ctc_03_asme1_nx800_rc_nx.stp & $\mathrm{NX}$ & SPLM & $11 / 04 / 15$ & 6 & & 10 & 13 & & 29 & & & 2 & & & 2 \\
\hline nist_ctc_03_asme1_nx800_rc_th.stp & $\mathrm{NX}$ & Theorem & $11 / 10 / 15$ & 6 & & 10 & 13 & & 29 & & & 1 & & & 1 \\
\hline nist_ctc_04_asme1_ct5210_rd_ct242repr.stp & CATIA & CoreTech & $12 / 19 / 14$ & 8 & & 10 & 5 & 1 & 24 & & & 3 & & 1 & 4 \\
\hline nist_ctc_04_asme1_ct5210_rd_dk242repr.stp & CATIA & Datakit & $02 / 16 / 15$ & 8 & & 11 & 5 & 1 & 25 & & & 2 & 2 & 1 & 5 \\
\hline nist_ctc_04_asme1_nx800_rd_ct242repr.stp & $\mathrm{NX}$ & CoreTech & $02 / 18 / 15$ & 8 & & 10 & 5 & & 23 & & & 3 & 1 & & 4 \\
\hline nist_ctc_04_asme1_nx800_rd_nx.stp & $\mathrm{NX}$ & SPLM & $11 / 04 / 15$ & 8 & & 10 & 5 & & 23 & & & 5 & & & 5 \\
\hline nist_ctc_04_asme1_nX800_rd_th.stp & NX & Theorem & $11 / 10 / 15$ & 8 & & 10 & 5 & & 23 & & & 3 & & & 3 \\
\hline nist_ctc_05_asme1_ct5210_rd_ct242repr.stp & CATIA & CoreTech & $12 / 19 / 14$ & 4 & 2 & 6 & 10 & & 22 & & 2 & 4 & 4 & & 10 \\
\hline nist ctc 05 asme1 ct5210 rd dk242repr.stp & CATIA & Datakit & $02 / 16 / 15$ & 4 & 2 & 6 & 10 & & 22 & & 2 & 3 & 3 & & 8 \\
\hline nist_ctc_05_asme1_nx800_rd_ct242repr.stp & $\mathrm{NX}$ & CoreTech & $02 / 18 / 15$ & 2 & 2 & 6 & 10 & & 20 & & 2 & 4 & 5 & & 11 \\
\hline nist_ctc_05_asme1_nx800_rd_nx.stp & $\mathrm{NX}$ & SPLM & $11 / 04 / 15$ & 2 & 2 & 6 & 10 & 2 & 22 & & 2 & 4 & & 2 & 8 \\
\hline \multirow{3}{*}{ nist_ctc_05_asme1_nx800_rd_th.stp } & NX & Theorem & $11 / 10 / 15$ & 4 & 2 & 6 & 10 & & 22 & 2 & 2 & 4 & 4 & & 12 \\
\hline & & & Counts: & 149 & 55 & 280 & 299 & 39 & 800 & 38 & 55 & 100 & 107 & 39 & 329 \\
\hline & & & Percents: & & & & & & & & & & & & \\
\hline
\end{tabular}

DFS = Datum Feature Symbol, DTS = Datum Tag Symbol, DIM = Dimension, FCF = Feature Control Frame 
Appendix B.2: Percent Clean (\%) by PMI Category for Test Models )

\begin{tabular}{|c|c|c|c|c|c|c|c|c|c|c|c|c|c|c|c|c|}
\hline STEP Model File & $\begin{array}{l}\text { Native } \\
\text { CAD }\end{array}$ & $\begin{array}{l}\text { STEP } \\
\text { Vendor }\end{array}$ & Date & $\begin{array}{l}\text { DFS } \\
\text { Count }\end{array}$ & $\begin{array}{c}\text { DTS } \\
\text { Count }\end{array}$ & $\begin{array}{c}\text { DIM } \\
\text { Count }\end{array}$ & $\begin{array}{l}\text { FCF } \\
\text { Count }\end{array}$ & $\begin{array}{l}\begin{array}{l}\text { NOTE } \\
\text { Count }\end{array} \\
\end{array}$ & $\begin{array}{l}\text { Total } \\
\text { Count }\end{array}$ & $\begin{array}{c}\text { DFS } \\
\text { Clean }\end{array}$ & $\begin{array}{c}\text { DTS } \\
\text { Clean }\end{array}$ & $\begin{array}{l}\text { DIM } \\
\text { Clean }\end{array}$ & $\begin{array}{l}\text { FCF } \\
\text { Clean }\end{array}$ & $\begin{array}{l}\text { NOTE } \\
\text { Clean }\end{array}$ & $\begin{array}{l}\text { Total } \\
\text { Clean }\end{array}$ & $\begin{array}{l}\text { Clean } \\
\text { Perc }\end{array}$ \\
\hline 827-9999-903.stp & $\mathrm{NX}$ & CoreTech & $04 / 28 / 15$ & 2 & & 8 & 6 & 7 & 23 & 2 & 0 & 3 & 6 & 0 & 11 & $48 \%$ \\
\hline 827-9999-904.stp & $\mathrm{NX}$ & CoreTech & $07 / 10 / 15$ & 3 & & 54 & 13 & 8 & 78 & 3 & 0 & 53 & 13 & 0 & 69 & $88 \%$ \\
\hline nist_ctc_01_asme1_ct5210_rd_ct242repr.stp & CATIA & CoreTech & $12 / 19 / 14$ & 3 & & 10 & 6 & 3 & 22 & 2 & 0 & 7 & 6 & 0 & 15 & $68 \%$ \\
\hline nist_ctc_01_asme1_ct5210_rd_dk242repr.stp & CATIA & Datakit & $02 / 16 / 15$ & 3 & & 10 & 6 & 3 & 22 & 3 & 0 & 6 & 6 & 0 & 15 & $68 \%$ \\
\hline nist_ctc_01_asme1_nx800_rd_ct242repr.stp & $\mathrm{NX}$ & CoreTech & $02 / 18 / 15$ & 3 & & 10 & 6 & 2 & 21 & 3 & 0 & 7 & 6 & 0 & 16 & $76 \%$ \\
\hline nist_ctc_01_asme1_nx800_rd_nx.stp & $\mathrm{NX}$ & SPLM & $11 / 04 / 15$ & 3 & & 10 & 6 & 2 & 21 & 3 & 0 & 1 & 6 & 0 & 10 & $48 \%$ \\
\hline nist_ctc_01_asme1_nx800_rd_th.stp & $\mathrm{NX}$ & Theorem & $11 / 10 / 15$ & 3 & & 10 & 6 & 2 & 21 & 3 & 0 & 9 & 6 & 0 & 18 & $86 \%$ \\
\hline nist_ctc_02_asme1_ct5210_rc_ct242repr.stp & CATIA & CoreTech & $12 / 19 / 14$ & 10 & 9 & 8 & 22 & $\frac{2}{1}$ & 50 & 0 & 0 & 0 & 0 & 0 & 0 & $0 \%$ \\
\hline nist_ctc_02_asme1_ct5210_rc_dk242repr.stp & CATIA & Datakit & $02 / 16 / 15$ & 10 & 9 & 8 & 22 & 1 & 50 & 0 & 0 & 0 & 0 & 0 & 0 & $0 \%$ \\
\hline nist_ctc_02_asme1_nx800_rc_ct242repr.stp & $\mathrm{NX}$ & CoreTech & $02 / 18 / 15$ & 7 & 9 & 7 & 22 & 1 & 46 & 0 & 0 & 0 & 0 & 0 & 0 & $0 \%$ \\
\hline nist_ctc_02_asme1_nx800_rc_nx.stp & $\mathrm{NX}$ & SPLM & $11 / 04 / 15$ & 6 & 9 & 7 & 22 & 2 & 46 & $\frac{6}{6}$ & 0 & 7 & 22 & 0 & 35 & $76 \%$ \\
\hline nist_ctc_02_asme1_nx800_rc_th.stp & $\mathrm{NX}$ & Theorem & $11 / 10 / 15$ & 10 & 9 & 7 & 22 & 1 & 49 & 0 & 0 & 0 & 0 & 0 & 0 & $0 \%$ \\
\hline nist_ctc_03_asme1_ct5210_rc_ct242repr.stp & CATIA & CoreTech & $12 / 19 / 14$ & 6 & & 10 & 13 & 1 & 30 & 6 & 0 & 8 & 13 & 0 & 27 & $90 \%$ \\
\hline nist_ctc_03_asme1_ct5210_rc_dk242repr.stp & CATIA & Datakit & $02 / 16 / 15$ & 6 & & 10 & 13 & 1 & 30 & 6 & 0 & 8 & 13 & 0 & 27 & $90 \%$ \\
\hline nist_ctc_03 asme1_nx800_rc_ct242repr.stp & NX & CoreTech & $02 / 18 / 15$ & 6 & & 10 & 13 & & 29 & 6 & 0 & 8 & 13 & 0 & 27 & $93 \%$ \\
\hline nist_ctc_03_asme1_nx800_rc_nx.stp & $\mathrm{NX}$ & SPLM & $11 / 04 / 15$ & 6 & & 10 & 13 & & 29 & 6 & 0 & 8 & 13 & 0 & 27 & $93 \%$ \\
\hline nist_ctc_03_asme1_nx800_rc_th.stp & $\mathrm{NX}$ & Theorem & $11 / 10 / 15$ & 6 & & 10 & 13 & & 29 & 6 & 0 & 9 & 13 & 0 & 28 & $97 \%$ \\
\hline nist_ctc_04_asme1_ct5210_rd_ct242repr.stp & CATIA & CoreTech & $12 / 19 / 14$ & 8 & & 10 & 5 & 1 & 24 & 8 & 0 & 7 & 5 & 0 & 20 & $83 \%$ \\
\hline nist_ctc_04_asme1_ct5210_rd_dk242repr.stp & CATIA & Datakit & $02 / 16 / 15$ & 8 & & 11 & 5 & 1 & 25 & 8 & 0 & 9 & 3 & 0 & 20 & $80 \%$ \\
\hline nist_ctc_04_asme1_nx800_rd_ct242repr.stp & $\mathrm{NX}$ & CoreTech & $02 / 18 / 15$ & 8 & & 10 & 5 & & 23 & 8 & 0 & 7 & 4 & 0 & 19 & $83 \%$ \\
\hline nist_ctc_04_asme1_nx800_rd_nx.stp & $\mathrm{NX}$ & SPLM & $11 / 04 / 15$ & 8 & & 10 & 5 & & 23 & 8 & 0 & 5 & 5 & 0 & 18 & $78 \%$ \\
\hline nist_ctc_04_asme1_nx800_rd_th.stp & $\mathrm{NX}$ & Theorem & $11 / 10 / 15$ & 8 & & $\frac{10}{10}$ & 5 & & 23 & 8 & 0 & 7 & 5 & 0 & 20 & $87 \%$ \\
\hline nist_ctc_05_asme1_ct5210_rd_ct242repr.stp & CATIA & CoreTech & $12 / 19 / 14$ & 4 & 2 & 6 & 10 & & 22 & 4 & 0 & 2 & 6 & 0 & 12 & $55 \%$ \\
\hline nist_ctc_05_asme1_ct5210_rd_dk242repr.stp & CATIA & Datakit & $02 / 16 / 15$ & 4 & 2 & 6 & 10 & & 22 & 4 & 0 & 3 & 7 & 0 & 14 & $64 \%$ \\
\hline nist ctc 05 asme 1 nx800 rd ct242repr.stp & $\mathrm{NX}$ & CoreTech & $02 / 18 / 15$ & 2 & 2 & 6 & 10 & & 20 & 2 & 0 & 2 & 5 & 0 & 9 & $45 \%$ \\
\hline \multirow{4}{*}{$\begin{array}{l}\text { nist_ctC_05_asme I_nxo0_ra_nX.stp } \\
\text { nist_ctc_05_asme1_nx800_rd_th.stp }\end{array}$} & $\mathrm{NX}$ & SPLM & $11 / 04 / 15$ & 2 & 2 & 6 & 10 & 2 & 22 & 2 & 0 & 2 & 10 & 0 & 14 & $64 \%$ \\
\hline & NX & Theorem & $11 / 10 / 15$ & 4 & 2 & 6 & 10 & & 22 & 2 & 0 & 2 & 6 & 0 & 10 & $45 \%$ \\
\hline & & & Counts: & 149 & 55 & 280 & 299 & 39 & 800 & 107 & 0 & 178 & 186 & 0 & 471 & $59 \%$ \\
\hline & & & Percents: & & & & & & & $72 \%$ & $0 \%$ & $64 \%$ & $62 \%$ & $0 \%$ & $59 \%$ & \\
\hline
\end{tabular}

DFS = Datum Feature Symbol, DTS = Datum Tag Symbol, DIM = Dimension, FCF = Feature Control Frame 